\title{
Intermittent fasting promotes adipose thermogenesis and metabolic homeostasis via VEGF-mediated alternative activation of macrophage
}

Kyoung-Han Kim ${ }^{1,12, *}$, Yun Hye Kim ${ }^{2, *}$, Joe Eun Son ${ }^{1}$, Ju Hee Lee ${ }^{2,3}$, Sarah Kim², Min Seon Choe ${ }^{1}$, Joon Ho Moon ${ }^{2}$ Jian Zhong ${ }^{2}$, Kiya Fu², Florine Lenglin², Jeong-Ah Yoo ${ }^{2}$, Philip J Bilan ${ }^{4}$, Amira Klip ${ }^{4}$, Andras Nagy ${ }^{5,6}$, Jae-Ryong Kim ${ }^{7}$, Jin Gyoon Park ${ }^{8}$, Samer MI Hussein ${ }^{9}$, Kyung-Oh Doh ${ }^{10}$, Chi-chung Hui ${ }^{11}$, Hoon-Ki Sung 2,3

${ }^{I}$ Developmental \& Stem Cell Biology Program, The Hospital for Sick Children, Toronto, Ontario, Canada, ${ }^{2}$ Translational Medicine Program, The Hospital for Sick Children, Toronto, Ontario M5G 1X8, Canada $;{ }^{3}$ Department of Laboratory Medicine and Pathobiology, University of Toronto, Toronto, Ontario M5S 1A1, Canada $;{ }^{4}$ Cell Biology Program, The Hospital for Sick Children, Toronto, Ontario, Canada; ${ }^{5}$ Lunenfeld-Tanenbaum Research Institute, Mount Sinai Hospital, Toronto, Ontario, Canada; ${ }^{6}$ Department of Obstetrics \& Gynaecology and Institute of Medical Science, University of Toronto, Toronto, Ontario, Canada; ${ }^{7}$ Department of Biochemistry and Molecular Biology, Smart-aging Convergence Research Center, College of Medicine, Yeungnam University, Daegu, Republic of Korea; ${ }^{8}$ Virginia G. Piper Center for Personalized Diagnostics, Biodesign Institute, Arizona State University, Tempe, AZ, USA; ' Centre Hospitalier Universitaire de Québec Research Center and Faculty of Medicine, Laval University, Quebec City, Quebec, Canada; ${ }^{10}$ Department of Physiology, College of Medicine, Yeungnam University, Daegu, Republic of Korea; ${ }^{11}$ Department of Molecular Genetics, University of Toronto, Toronto, Ontario M5S 1A1, Canada

Intermittent fasting (IF), a periodic energy restriction, has been shown to provide health benefits equivalent to prolonged fasting or caloric restriction. However, our understanding of the underlying mechanisms of IF-mediated metabolic benefits is limited. Here we show that isocaloric IF improves metabolic homeostasis against diet-induced obesity and metabolic dysfunction primarily through adipose thermogenesis in mice. IF-induced metabolic benefits require fasting-mediated increases of vascular endothelial growth factor (VEGF) expression in white adipose tissue (WAT). Furthermore, periodic adipose-VEGF overexpression could recapitulate the metabolic improvement of IF in non-fasted animals. Importantly, fasting and adipose-VEGF induce alternative activation of adipose macrophage, which is critical for thermogenesis. Human adipose gene analysis further revealed a positive correlation of adipose VEGF-M2 macrophage-WAT browning axis. The present study uncovers the molecular mechanism of IF-mediated metabolic benefit and suggests that isocaloric IF can be a preventive and therapeutic approach against obesity and metabolic disorders.

Keywords: intermittent fasting; thermogenesis; vascular endothelial growth factor; adipose macrophage Cell Research (2017) 27:1309-1326. doi:10.1038/cr.2017.126; published online 17 October 2017

\section{Introduction}

\footnotetext{
*These two authors contributed equally to this work. Correspondence: Chi-chung Hui ${ }^{\mathrm{a}}$, Hoon-Ki Sung ${ }^{\mathrm{b}}$

å-mail: cchui@sickkids.ca

${ }^{\mathrm{b}}$ E-mail: hoon-ki.sung@sickkids.ca

${ }^{12}$ Current address: University of Ottawa Heart Institute and Department of Cellular and Molecular Medicine, Faculty of Medicine, University of Ottawa, Ottawa, Ontario, Canada

Received 3 March 2017; revised 30 June 2017; accepted 26 July 2017; published online 17 October 2017
}

While fat (white adipose tissue, WAT) is often associated with development of obesity and type 2 diabetes, it is essential for energy homeostasis by storing excess energy and releasing lipids in response to energy deficits [1, 2]. Recent studies have discovered that WAT also contributes to whole-body metabolism by regulating thermogenic activity via the browning of WAT, which increases energy expenditure and improves insulin sensitivity [3]. In this regard, WAT browning has been suggested as a therapeutic approach for obesity and metabolic diseases. 
A variety of physiological stimuli (e.g., cold exposure, exercise) and signaling ligands (e.g., FGF21, BMP, VEGF) have been identified for their potential to induce WAT browning [4]. In addition, recent studies highlight the critical role of the innate immune response with alternative activation of adipose macrophage (M2 macrophage) in the regulation of metabolic function of adipose tissues, including WAT browning [5].

We and others have previously demonstrated that vascular endothelial growth factor in adipose tissues (adipose-VEGF) plays a key role in browning of WAT and maintaining the functional integrity of adipose tissues, suggesting the therapeutic potential of VEGF in the treatment of metabolic disorders [6-9]. For example, cold acclimatization [10], exercise [11], and environmental stimuli [9] promote the expression of adipose-VEGF and hence WAT browning. However, the molecular mechanism underlying VEGF-mediated WAT browning remains elusive.

Modern lifestyles favor longer periods of daily energy intake and shorter fasting periods. This erratic eating pattern is associated with metabolic disadvantages and contributes to the current global obesity and diabetes epidemic [12]. Fasting brings various positive health impacts, suggesting that modulation of fasting period can be used as a therapeutic intervention [13, 14]. Intermittent fasting (IF), a periodic energy restriction method, has been shown to provide health benefits equivalent to prolonged fasting or caloric restriction (CR) [14-18]. The beneficial effects of IF against aging, cancer, cardiovascular diseases, and neurodegenerative diseases have been studied in both animal models and clinical settings $[15,19-21]$. However, it is unclear whether the metabolic benefits conferred by IF are primarily mediated by reduced food intake $[19,22,23]$ or attributed to changes in eating pattern. In particular, the 'alternate day fasting' regimen (i.e., 1 day feeding-1 day fasting, 1:1 IF), which is often used in rodent IF models, could result in underfeeding $[19,24]$. In addition, while WAT is one of the most critical organs for fasting physiology, it is not known whether the molecular alteration of WAT after IF is a driver or an outcome of IF-mediated metabolic benefits.
In this study, we establish a new IF regimen to investigate the impact of IF under isocaloric conditions and demonstrate that IF improves glucose homeostasis and prevents diet-induced metabolic dysfunction without caloric intake reduction. Mechanistically, we found that WAT is pivotal for mediating IF-induced metabolic benefits via browning of WAT through adipose-VEGF-mediated alternative activation of adipose macrophage (M2 macrophage). Our study unveils a novel mechanism by which IF promotes whole-body homeostasis through browning of WAT by VEGF-mediated macrophage switching.

\section{Results}

\section{An IF regimen protects mice from diet-induced metabolic} abnormalities

To minimize the differences in caloric intake which may be caused by the alternate day fasting [19], we developed a new IF regimen comprising 2 day feeding- 1 day fasting periods (2:1 IF; Figure 1A). This regimen provided mice with sufficient time to compensate for the decreased body weight and for the reduced amount of food intake after 1-day fasting, to the level of non-fasted animals, enabling us to examine the effects of IF, independent of caloric intake difference (Supplementary information, Figure S1A-S1C).

Eight-week-old mice were subjected to 16 weeks of the 2:1 IF regimen on either normal chow diet (ND) or $45 \%$ high-fat diet (HFD). Compared to mice fed ad libitum (AL), IF mice showed lower body weight on both ND and HFD (Figure 1B; Supplementary information, Figure S1D and S1E). In further analyses, we focused mainly on IF and AL mice fed HFD (HFD-IF and HFDAL) because they showed a marked difference in weight gain than those fed ND (Supplementary information, Figure S1F). We speculated that the reduced weight gain of IF animals might be due to the slight decrease in accumulated energy intake over 16 weeks of the diet program (Figure 1C). However, our pair-feeding experiments consistently showed that IF animals exhibited reduced weight gain, compared to mice pair-fed ad libitum with

Figure $1 \mathrm{IF}$ protects mice from diet-induced obesity and metabolic dysfunction. (A) Schematic illustration of the 2:1 IF regimen. (B) Body weight measurement during 16 weeks of IF. (C) Changes of energy intake during 16 weeks of IF cycles. The inset shows total energy intake during 16 weeks of IF cycles. (D) Body composition showing fat and lean mass. (E) H\&Estained sections of adipose tissues; IWAT (subcutaneous), PWAT (visceral), and BAT. (F) Average of cross-sectioned area of subcutaneous white adipocytes, revealing reduced white adipocyte size for HFD-IF mice compared to HFD-AL mice. (G) GTT in HFD-AL and HFD-IF mice. The inset shows AUC. (H) ITT. The inset shows AUC. (I) HOMA-IR. Data are expressed as mean \pm SEM (ND-AL: $n=7$; ND-IF: $n=8$; HFD-AL: $n=7$; and HFD-IF: $n=8$ ); one or two-way ANOVA with Student-Newman-Keuls post hoc analysis and two-tailed unpaired Student's $t$-test; ${ }^{*} P<0.05$ and ${ }^{* *} P<0.01$ vs HFD-AL. AL, ad libitum; AUC, area under the curve; HFD, high-fat diet; ND, normal diet. 
A

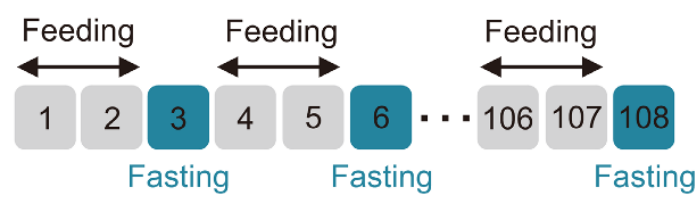

ND-AL : Normal chow, ad-libitum

ND-IF : Normal chow, intermittent fasting

HFD-AL : High-fat diet, ad-libitum

HFD-IF : High-fat diet, intermittent fasting

C

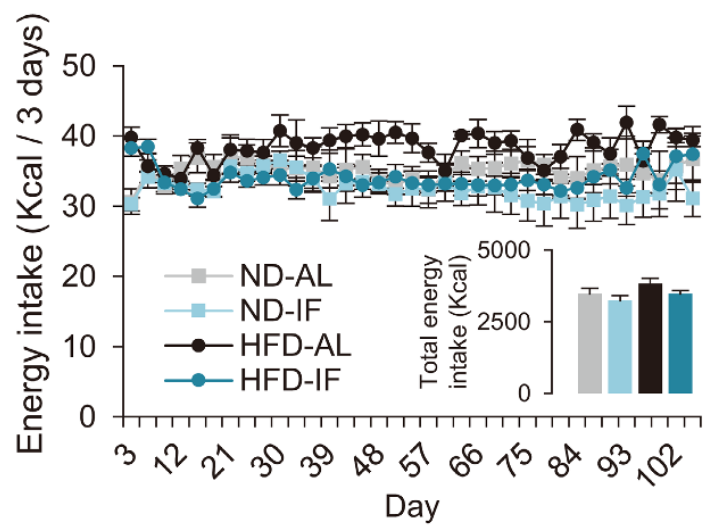

E

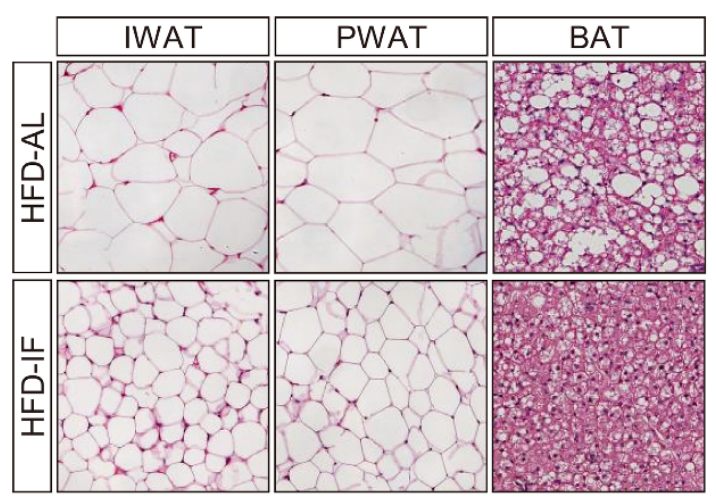

B
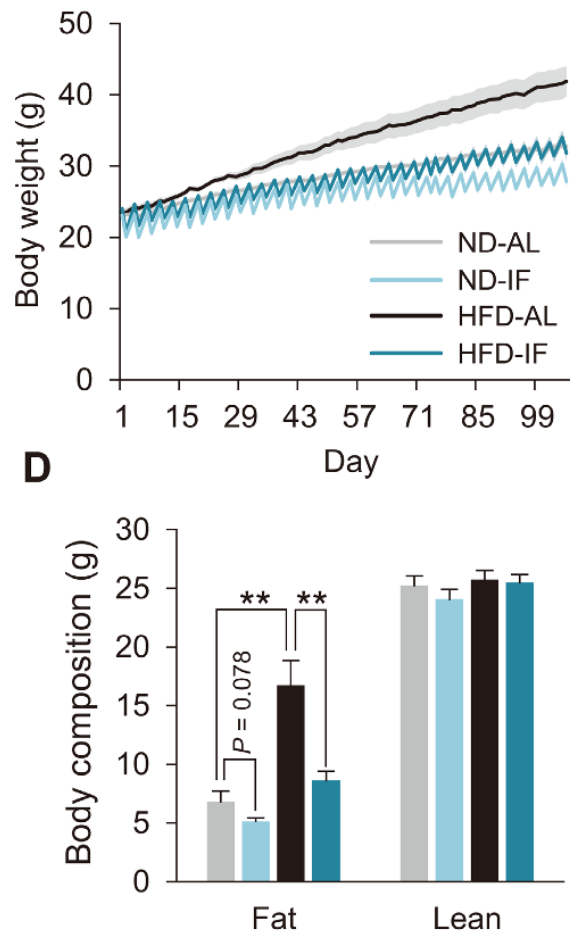

$\mathbf{F}$

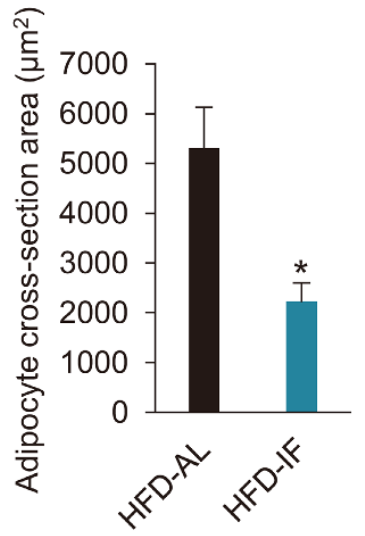

G

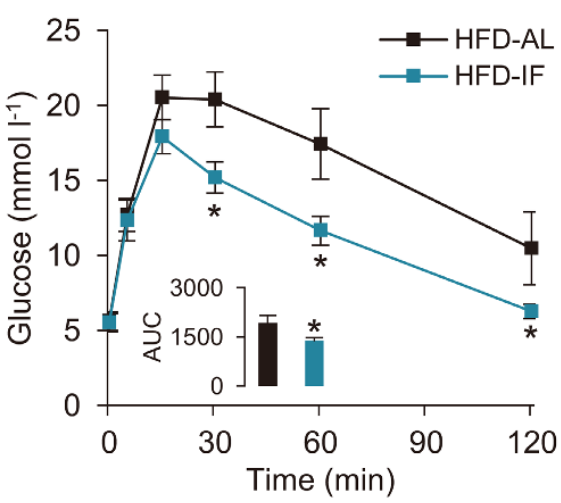

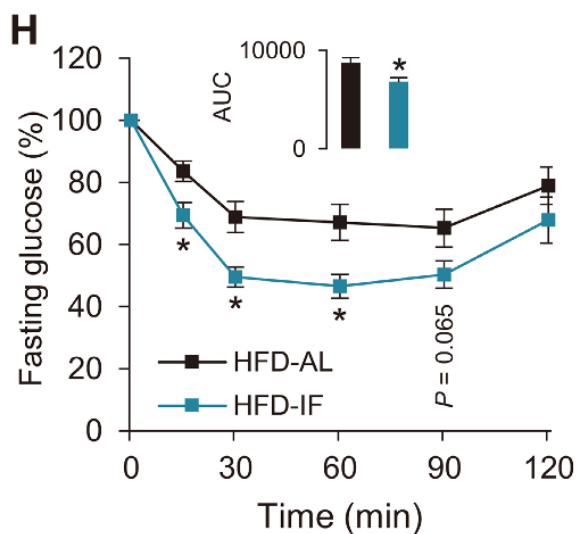

I

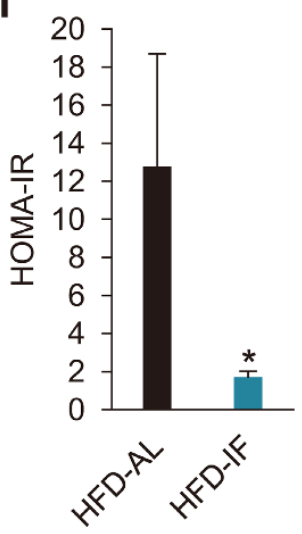


exactly the same amount of food as IF mice (HFD-PF; Supplementary information, Figure S2A-S2C). These data suggest that IF-mediated decrease in body weight is not primarily attributed to an energy intake difference. Body composition analysis revealed reduced fat mass without changes of lean mass in IF mice (Figure 1D and Supplementary information, Figure S2D). Consistently, HFD-IF mice exhibited a reduction in tissue weight and adipocyte size of both inguinal WAT (IWAT) and perigonadal WAT (PWAT) depots, compared to HFDAL mice (Figure 1E and 1F; Supplementary information, Figure $\mathrm{S} 1 \mathrm{G})$. Lipid accumulation in brown adipose tissue (BAT) was also decreased in HFD-IF, compared to HFDAL mice (Figure 1E). Notably, HFD-IF mice showed improved glucose homeostasis with smaller glucose excursion in glucose tolerance test (GTT), increased insulin sensitivity in insulin tolerance test (ITT), and markedly lower homeostasis model assessment-estimated insulin resistance (HOMA-IR), compared to HFD-AL or HFDPF mice (Figure 1G-1I; Supplementary information, Figure S2E and S2F). Moreover, IF prevented HFD-induced hepatic steatosis. IF mice exhibited lower liver weight, less lipid accumulation in liver, and lower plasma alanine aminotransferase (ALT) activity (Supplementary information, Figure S1H-S1L). Together, these findings demonstrate that, in the absence of any energy intake difference, IF offers metabolic benefits against diet-induced obesity and metabolic dysfunction.

We also tested the therapeutic potential of IF on established metabolic abnormalities by performing $2: 1$ IF on obese mice induced by HFD for 12 weeks (post HFD; Supplementary information, Figure S3A) and were able to observe beneficial effects as early as 6 weeks of IF. This short-term 2:1 IF treatment on post HFD mice resulted in a slight reduction in body weight without changes in total caloric intake (Supplementary information, Figure S3B and S3C). Consistent with our results above, IF led to a selective decrease in fat mass, particularly in WAT mass and adipocyte size, whereas lean mass was not affected (Supplementary information,
Figure S3D-S3F). Liver function parameters were also improved by 6 weeks of IF (Supplementary information, Figure S3G-S3J). Importantly, short-term IF treatment significantly improved systemic glucose homeostasis as assessed by GTT and HOMA-IR, compared to untreated mice (Supplementary information, Figure S3K and S3L). Together, these data indicate that IF is not only protective method, but also effective therapeutics against obesity.

\section{Transcriptome analysis of IF-treated WAT}

It has been suggested that IF promotes various health benefits, yet the underlying mechanism is still not fully understood. Given that adipose tissues are pivotal for supplying energy to other tissues during fasting, and were one of the most affected organs/tissues after IF, we performed transcriptome analysis by RNA sequencing (RNA-seq) on PWAT from mice subjected to IF or AL to gain insight into the genome-wide alteration of adipose tissue gene expression by IF. Excluding HFD-AL, the ND-AL, ND-IF and HFD-IF groups clustered together, indicating that even with HFD feeding, IF induced a global change in transcriptome that resembles gene expression profiles of metabolically normal animals (Figure 2A). Overall, 3644 genes were differentially expressed among the four conditions and formed 6 distinct gene clusters. Clusters 1, 3, and 4 demonstrated significant gene enrichment by using terms of gene ontology (GO term) and KEGG pathway databases (Figure 2A; Supplementary information, Figure S4 and Table S1). In particular, we focused on clusters 1 and 3 that show dramatic differences in gene expression profile between HFD-AL and HFD-IF with clearly distinguished metabolic phenotypes. Cluster 1 genes (i.e., upregulated specifically in HFD-AL compared to other groups) were strongly enriched in the pathways involved in the immune response and inflammation, which are associated with insulin resistance and metabolic dysfunction [25] (Figure 2A and Supplementary information, Figure S4A). qPCR verified that genes associated with inflammatory cells and their chemotactic activity were significantly downregulated in

Figure 2 Transcriptome analysis upon IF reveals browning of WAT. (A) Heatmap displaying 3644 differentially expressed genes among ND-AL, ND-IF, HFD-AL, and HFD-IF groups that were clustered into six distinct gene groups. Enriched GO terms and representative genes are shown at the right for each cluster. Significantly enriched clusters: red boxes. Not significantly enriched clusters: gray boxes. (B) Gene expression analysis on inflammation-related genes. (C) Marker gene expression of sympathetic activation (i.e., Adrb3) and brown/beige adipocyte (i.e., Ppargc1a, Ppargc1b, Cidea and Ucp1) (HFD, AL/IF: $n=6 / 8$ ). (D) Representative images of whole-mount WAT of the brown/beige chaser (Ucp1-Cre;Rosa26 $6^{\mathrm{mT} / \mathrm{mG}}$ ) $\mathrm{mice}$ subjected to AL and IF. Beige adipocytes are visualized by membrane-targeted GFP (mG; green). (E) $\mathrm{O}_{2}$ consumption during fasting and feeding condition. $(F)$ Linear regression analysis of $\mathrm{O}_{2}$ consumption as a function of body weight during feeding period. The inset shows $\mathrm{O}_{2}$ consumption during feeding condition adjusted with body weight at $38.54 \mathrm{~g}$ using ANCOVA (HFD, AL/IF: $n=6 / 12$ ). (G) Significant changes in $\mathrm{O}_{2}$ consumption between fasting and feeding periods in HFD-IF mice. Values are mean \pm SEM; two-tailed unpaired and paired Student's $t$-test; ${ }^{*} P<0.05$ vs HFD-AL. 

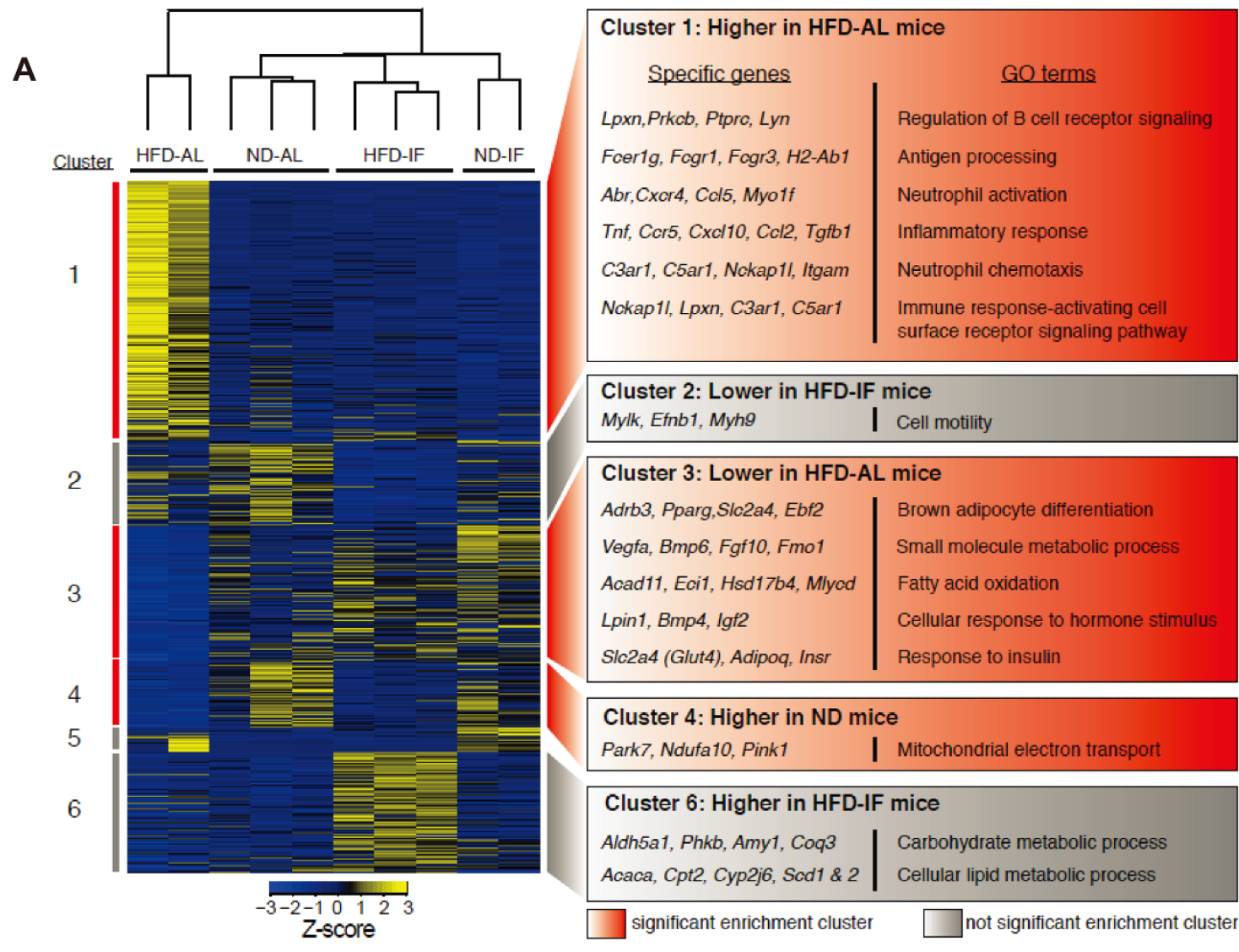

Cluster 2: Lower in HFD-IF mice

Mylk, Efnb1, Myh9 I Cell motility

Cluster 3: Lower in HFD-AL mice

Adrb3, Pparg,Slc2a4, Ebf2

Vegfa, Bmp6, Fgf10, Fmo1

Acad11, Eci1, Hsd17b4, Mlycd

Lpin1, Bmp4, Igf2

Slc2a4 (Glut4), Adipoq, Insr

\begin{tabular}{|l|l|}
\hline Cluster 4: Higher in ND mice \\
Park7, Ndufa10, Pink1 I Mitochondrial electron transport
\end{tabular}

\begin{tabular}{|l|l|}
\hline $\begin{array}{l}\text { Cluster 6: Higher in HFD-IF mice } \\
\text { Aldh5a1, Phkb, Amy1, Coq3 }\end{array}$ & $\begin{array}{l}\text { Carbohydrate metabolic process } \\
\text { Cellular lipid metabolic process }\end{array}$ \\
\hline Acaca, Cpt2, Cyp2j6, Scd1 \& 2 & significant enrichment cluster $\quad \square$ not significant enrichment cluster
\end{tabular}

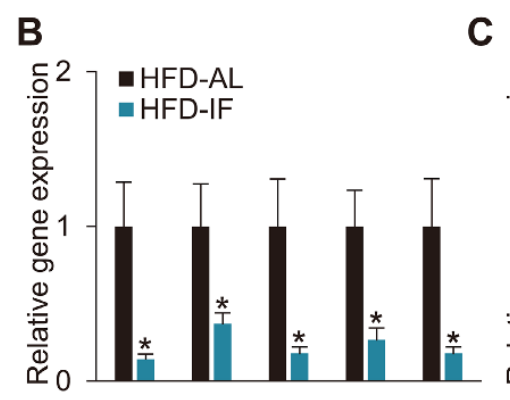

C

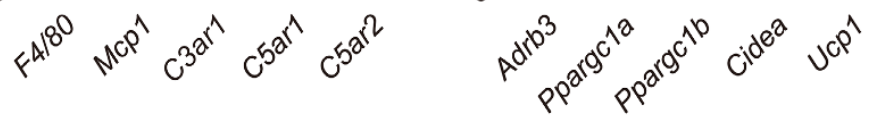
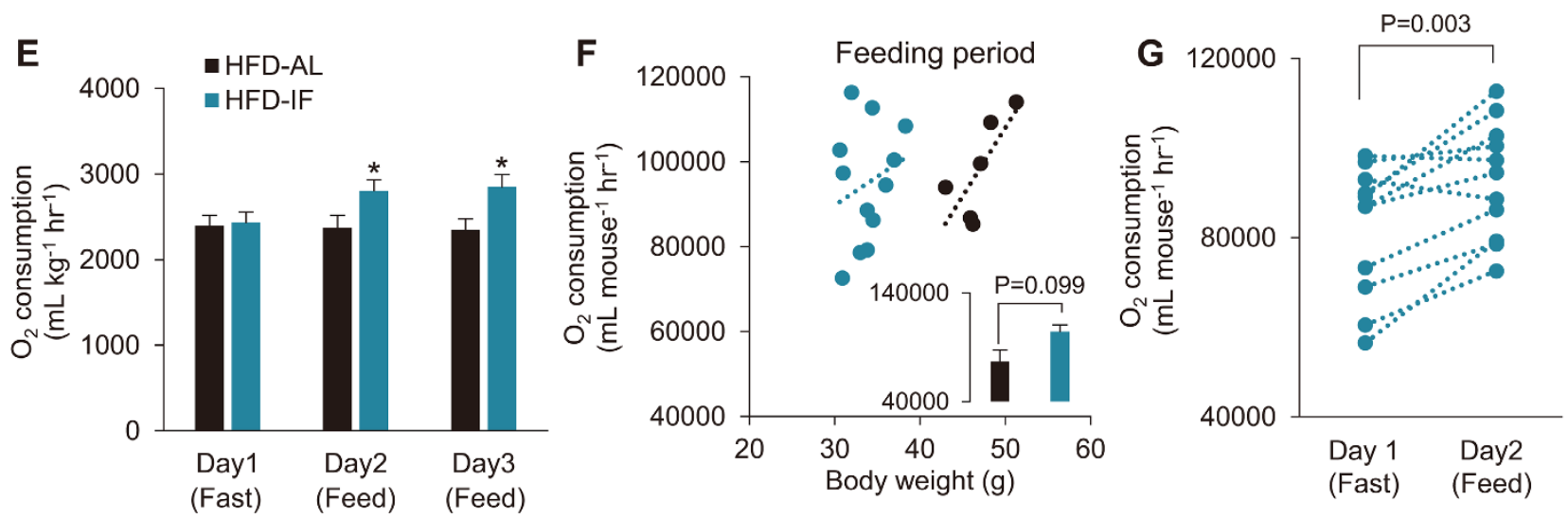
HFD-IF compared to HFD-AL (Figure 2B). On the other hand, GO term analysis of cluster 3 genes (i.e., downregulated specifically in HFD-AL) revealed biological processes including brown adipocyte differentiation (i.e., Ebf2) [26] (Figure 2A and Supplementary information, Figure S4B). This finding is aligned with cluster 6 (i.e., upregulated only in HFD-IF group) indicating that the futile metabolic cycle with concurrently elevated genes involved in fatty acid synthesis (e.g., Acaca, Scdl and $S c d 2$ ) and oxidation (e.g., Cpt2), as seen in $\beta 3$-adrenergic receptor ( $\beta 3$-AR) agonist CL-316243- and cold-induced beige adipocytes $[27,28]$. Together, these results suggest that IF promotes a reduction of inflammation and an increase of thermogenic activities in WAT.

\section{IF leads to adipose thermogenesis}

Next, we validated browning of WAT in HFD-IF mice. The expression of $A d r b 3$ (encoding 33 -AR; indicative of sympathetic activation) as well as levels of beige/ brown adipose markers (i.e., Ppargcla, Cidea and Ucp 1) were significantly elevated in PWAT of HFD-IF mice, compared to that of HFD-AL mice (Figure 2C). Similar results were also observed in WAT of post HFD-IF mice (Supplementary information, Figure S5A). In addition, Ucpl gene expression in BAT was found to be higher in HFD-IF mice compared to HFD-AL mice (Supplementary information, Figure S5B), suggesting increased BAT activation by IF. To visually examine beige adipocytes, we also utilized the beige-chaser mice generated by crossing Ucp 1-Cre with Rosa $26^{m T / m G}$ mice. HFDIF WAT exhibited more $\mathrm{GFP}^{+}$beige adipocytes (Figure 2D), whereas the number of $\mathrm{GFP}^{+}$brown adipocytes in BAT was indistinguishable between HFD-IF and HFDAL mice (Supplementary information, Figure S5C). Consistent with the elevated level of beige adipocytes and higher Ucpl expression, indirect calorimetry, with normalization of body mass or adjustment for body mass using regression-based analysis of covariance (ANCOVA), revealed that $\mathrm{O}_{2}$ consumption in HFD-IF mice was increased, particularly during the feeding period (i.e., Day 2), without significant changes in physical activities, compared to HFD-AL mice (Figure 2E-2G; Supplementary information, Figure S5D-S5G). On the other hand, $\mathrm{O}_{2}$ consumption during the fasting period (i.e., Day 1) was not different between groups (Figure 2E and Supplementary information, Figure S5E). Together, these data suggest that IF led to an increase in beige adipocytes, thereby elevating energy expenditure, particularly during energy intake.

Adipose thermogenesis largely contributes to IF-mediated metabolic benefits
To examine whether the beneficial effects of IF could be attributed to adipose thermogenesis, we performed IF in a thermoneutral $(\mathrm{TN})$ condition $\left(30^{\circ} \mathrm{C}\right)$, where the thermogenic activation is significantly restricted [29]. Indeed, despite increased sympathetic activation (i.e., higher $A d r b 3$ expression), TN-HFD-IF mice showed no significant changes of beige/brown adipose marker gene expression in PWAT, compared to TN-HFD-AL mice (Supplementary information, Figure S6A). This suggests that thermoneutrality effectively hindered IF-induced WAT browning. Although Ucp 1 expression in BAT was increased by IF under TN conditions, its basal expression level was much lower $(\sim 10 \%)$ compared to that at normal temperature (Supplementary information, Figure $\mathrm{S} 6 \mathrm{~B})$. Consistently, $\mathrm{O}_{2}$ consumption was not significantly different between the two groups under $\mathrm{TN}$ feeding condition (Supplementary information, Figure S6C), suggesting that the increased Ucp1 in BAT of TN-HFDIF mice may not be functionally significant. Intriguingly, however, TN-HFD-IF mice still exhibited a mild improvement in glucose tolerance, insulin sensitivity, and HOMA-IR, with a reduction in body weight, fat mass, as well as hepatic steatosis, compared to TN-HFD-AL mice (Supplementary information, Figure S6D-S6L). Although the TN condition does not completely abolish the metabolic benefits of IF, it significantly reduced the degree of improvement by IF (e.g., GTT difference: $9 \%$ in TN vs $28 \%$ in normal temperature), further emphasizing the key contributing role of adipose thermogenesis in IF.

\section{Fasting induces adipose-VEGF expression}

We next aimed to identify a molecular driver of IF-induced metabolic benefits and adipose thermogenesis. As WAT plays a crucial role in whole-body metabolic homeostasis through the production of numerous secretory proteins including adipokines [30,31], we examined changes of the chemokine and adipokine pathways between HFD-AL and HFD-IF. Out of 710 adipose secretory proteins as defined by Lehr et al. [30], several potential adipokine genes were markedly upregulated in HFDIF PWAT, including Vegfa (VEGF), Cfd (adipsin), Nrg4 (neuregulin 4), and Adipoq (adiponectin), whereas Lep (leptin), which positively correlates with adiposity, was downregulated (Figure 3A and 3B). To test whether these genes potentially function as drivers or are simply outcomes of repeated fasting, we examined how one-time fasting affects the expression of these genes. Noticeably, a 24-h fasting significantly increased Vegfa expression in WAT, while reducing Lep and Nrg4 transcripts (Figure $3 \mathrm{C})$. Given that higher expression of VEGF, leptin and neuregulin 4 positively correlates with adipose thermogenesis $[8,9,32,33]$, these results suggest that VEGF in 


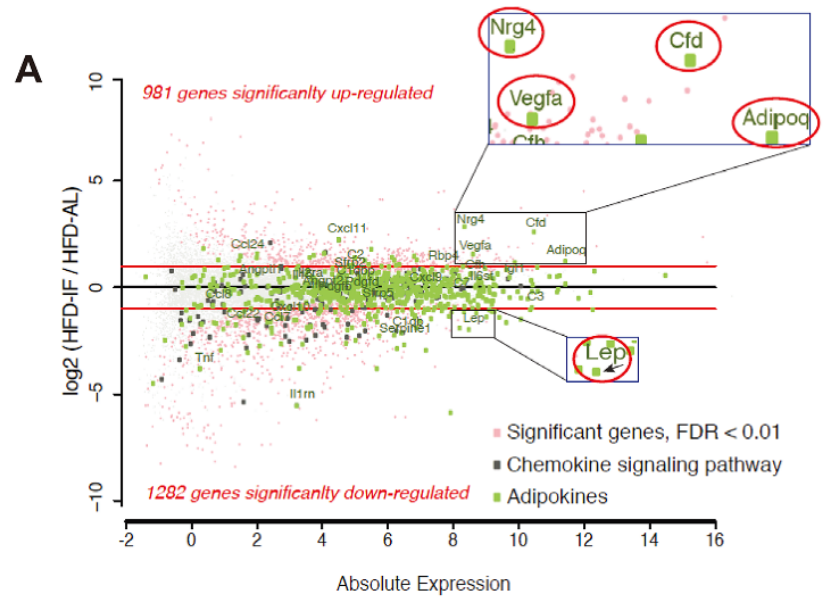

B

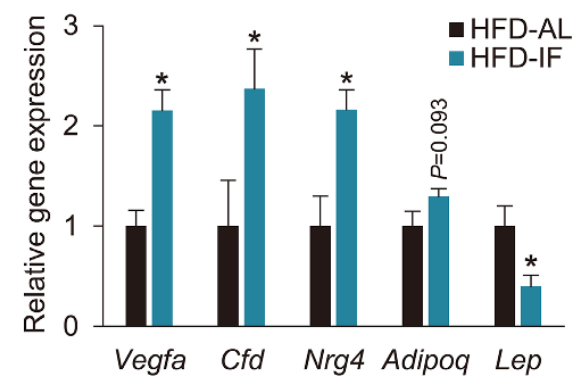

C

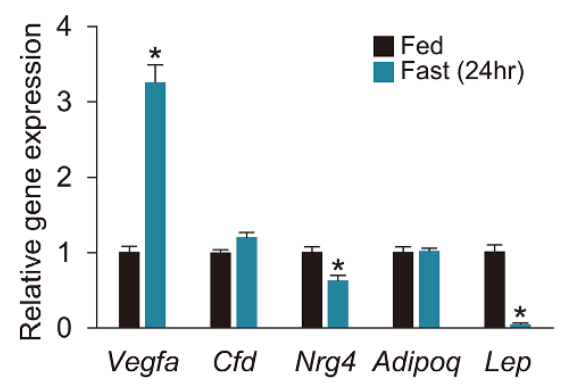

G

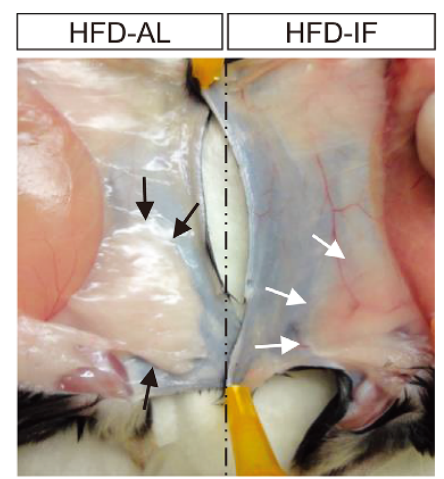

D

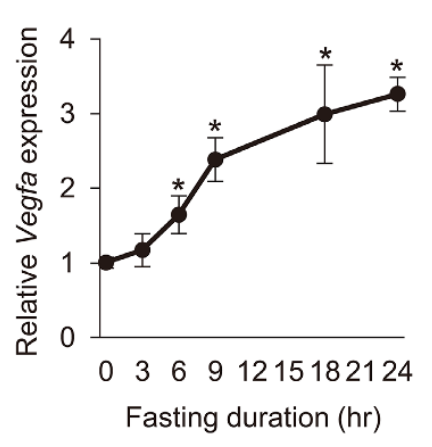

E

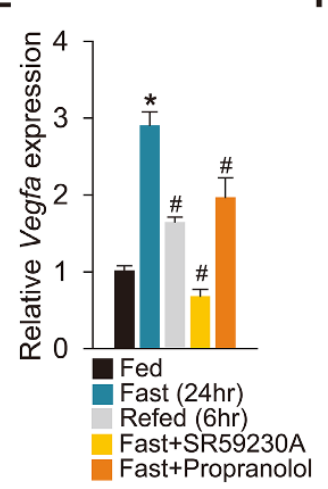

$\mathbf{F}$

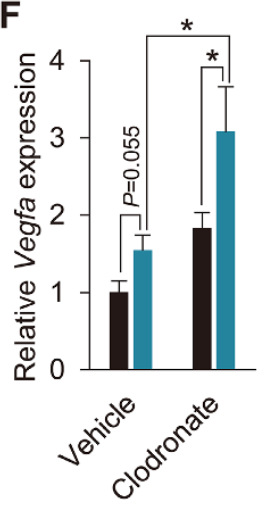

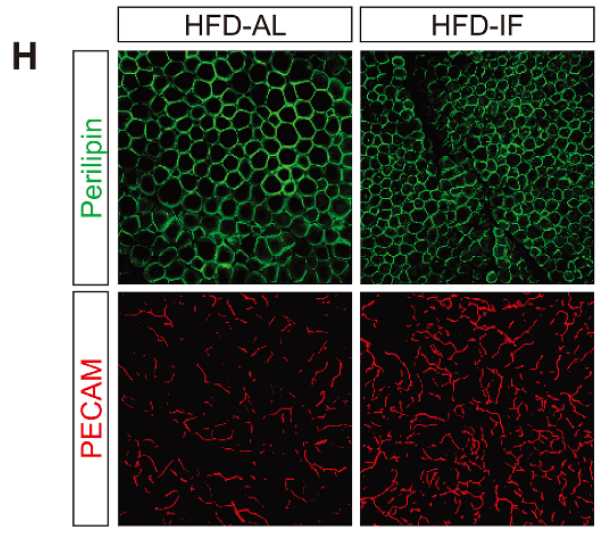

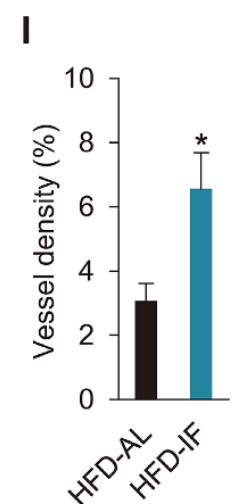

Figure 3 Fasting induces adipose-VEGF expression. (A) MA plot highlighting significantly altered mRNA expression of adipose-derived factors in PWAT of HFD-IF mice, compared to that of HFD-AL mice. Vegfa, Cfd, Nrg4, Adipoq, and Lep encode vascular endothelial growth factor, adipsin, neuregulin 4, adiponectin, and leptin, respectively. (B) qPCR validation of adipose-derived factors in PWAT (HFD, AL/IF: $n=6 / 8$ ). (C) mRNA expression levels of Vegfa, Cfd, Nrg4, Adipoq, and Lep in PWAT at feeding and $24 \mathrm{~h}$ of fasting $(n=5$ per group). (D) Vegfa mRNA expression in PWAT at different fasting durations $(n$ $=5-6$ per group). (E) Vegfa mRNA expression in PWAT at feeding, $24 \mathrm{~h}$ of fasting, $6 \mathrm{~h}$ of refeeding, fasting with $\beta 3-\mathrm{AR}$ antagonist, SR59230A (5 mg/kg, i.p.), and fasting with non-specific $\beta$-AR antagonist, Propranolol ( $5 \mathrm{mg} / \mathrm{kg}$, i.p.; $n=5$ per group). (F) Vegfa mRNA expression in PWAT at feeding and $24 \mathrm{~h}$ of fasting with treatments of vehicle or clodronate $(n=5)$. (G) A representative macroscopic image illustrating increased vascularization in IWAT of HFD-IF mice, compared to HFD-AL mice. Black and white arrows indicate IWAT of HFD-AL and HFD-IF mice, respectively. (H) Representative microscopic images of adipocytes and blood vessels, visualized with perilipin and PECAM-1 antibodies, respectively, in whole-mount PWAT. (I) Quantification of vessel densities in PWAT. Data are mean \pm SEM; one- or two-way ANOVA with Student-Newman-Keuls post hoc analysis and two-tailed unpaired Student's $t$-test; ${ }^{*} P<0.05$ vs HFD-AL or Fed. ${ }^{\#} P<0.05$ vs Fast $(24 \mathrm{~h})$. 
WAT, but not leptin or Nrg4 (both decreased by fasting), is a potential modulator of IF.

We further examined how and where fasting-induced VEGF expression is regulated. Vegfa expression levels in WAT increase progressively with the fasting duration and the increase can be immediately reversed by refeeding (Figure 3D and 3E). Importantly, fasting-stimulated VEGF expression is restricted to WAT, not in BAT, other metabolic tissues or plasma (Supplementary information, Figure S7A-S7D). While previous studies showed that sympathetic activity controls VEGF expression [34], total plasma catecholamine level was not increased by fasting (Supplementary information, Figure S7E), suggesting that fasting-stimulated VEGF expression in WAT is regulated via peripheral, not central, sympathetic nervous system. Moreover, fasting-induced VEGF was blocked markedly by the $\beta 3$-AR antagonist, SR59230A, but less effectively by the non-specific $\beta$-AR antagonist, propranolol (Figure 3E). Given that $\beta 3$-AR is known to be expressed specifically in adipocytes $[35,36]$, this result suggests that adipocytes may be the source of VEGF in response to sympathetic activation by fasting. To test this possibility, macrophages, which are another source of VEGF [37, 38], were depleted in mice by administration of Clodronate [37, 39]. Notably, macrophage depletion, verified by abolished $F 4 / 80$ gene expression (Supplementary information, Figure S7F), did not affect fasting-mediated VEGF induction in WAT and was even increased in expression levels compared to vehicle-treated control mice (Figure 3F). This finding further supports our conclusion that adipocytes are the major sources of fasting-mediated VEGF induction. Functionally, the acute increase of adipose-VEGF by a 24-h fasting was not sufficient to induce changes in vascularization (Supplementary information, Figure S7G), whereas repeated fasting (i.e., IF) significantly increased WAT vascularization, both macroscopically and microscopically (Figure 3G-3I). Given that improved vascularization with adipose-derived VEGF plays a positive role in adipose tissue function and metabolic homeostasis against obesity and diabetes [6-8], our results together suggest that fasting-induced adipose VEGF expression underlies IF-induced metabolic benefits and adipose thermogenesis.

\section{Adipose-VEGF is required for IF-mediated metabolic benefits}

We then investigated the implication of fasting-induced adipose-VEGF in the metabolic benefits of IF. Since VEGF generated by adipocytes and resident macrophages in adipose tissues could potentially influence and compensate each other, we utilized aP2-Cre mice that express Cre recombinase in preadipocytes, adipo- cytes, and macrophages [40] to generate pan-adipose Vegfa KO mice (aP2-Cre; Vegfa ${ }^{f l o x f f o x}$; hereafter VEGF ${ }^{\mathrm{AdKO}}$ ), and conducted IF with this loss-of-function mouse model. Intriguingly, IF had no significant effect on WAT weight and adipocyte size in VEGF ${ }^{\mathrm{AdKO}}$ mice fed HFD even with decreased body weight (Figure 4A-4C). Consistent with this, adipose Lep gene expression and plasma leptin levels, which positively correlate with adiposity, were also indistinguishable between VEGF ${ }^{\text {AdKO }}$-HFD-IF and VEGF $^{\text {AdKO }}$-HFD-AL mice (Figure 4D and 4E). These data suggest that IF reduced body weight at the expense of lean mass. In addition, GTT and HOMA-IR revealed that IF does not improve glucose homeostasis in VEG$\mathrm{F}^{\text {AdKO }}$ mice (Figure $4 \mathrm{~F}$ and $4 \mathrm{G}$ ). IF-mediated improvement of liver steatosis was also abolished in VEGF ${ }^{\mathrm{AdKO}}$ mice (Supplementary information, Figure S8A-S8C). Importantly, loss of adipose-VEGF abolished IF-mediated WAT browning, and there was no difference in Ucpl gene expression even in the presence of sympathetic activation (i.e., increased Adrb3; Figure 4H). Moreover, IF-induced BAT activation was not observed in VEG$\mathrm{F}^{\mathrm{AdKO}}$ mice (Figure $4 \mathrm{I}$ ). Together, these findings suggest that adipose-VEGF is required for IF-induced metabolic improvement and adipose thermogenesis.

Intermittent adipose-VEGF overexpression is sufficient to mimic the IF-mediated metabolic benefits

The results above prompted us to test whether periodic cycles of adipose-VEGF upregulation without fasting are sufficient to mimic the metabolic impact by IF. To mimic the fasting-mediated VEGF upregulation, two different lines of inducible adipose-VEGF mice (adipocytes + macrophage: aP2-Cre;Rosa2 $6^{r t T A} ;$ Tet-O-VEGF, hereafter $\mathrm{VEGF}^{\mathrm{aP2} 2 \mathrm{Tg}}$; adipocytes: Adipoq-Cre;Rosa2 $6^{\text {rt- }}$ ${ }^{T A}$;Tet-O-VEGF, hereafter $\mathrm{VEGF}^{\mathrm{Adipoq}-\mathrm{Tg}}$ ) were intermittently subjected to $45 \%$ HFD containing doxycycline (HFD-DOX), similar to IF cycle (Figure 5A). Importantly, both VEGF $^{\text {aP2-Tg }}$ and VEGF ${ }^{\text {Adipoq-Tg }}$ mice under HFDDOX cycles showed very similar metabolic phenotypes seen in HFD-IF mice, compared to control mice (VEG$\mathrm{F}^{\mathrm{CTRL}}$; Figure $5 \mathrm{~B}$ ). Both transgenic mice showed significantly elevated adipose Vegfa expression and vascularization (Figure 5C and 5D; Supplementary information, Figure S9A). They also exhibited lower body weight due to decreased fat mass without changes of lean mass, reduced adipose tissue weight and cell size, and improved glucose homeostasis (Figure 5E-5H). Moreover, both transgenic mouse lines showed browning of WAT with elevated Ucp1 and Cidea expression (Figure 5I), comparable to previous reports [6-8], without BAT activation (Supplementary information, Figure S9B). Together with the data of adipose-specific Vegfa KO mice, these results 
A

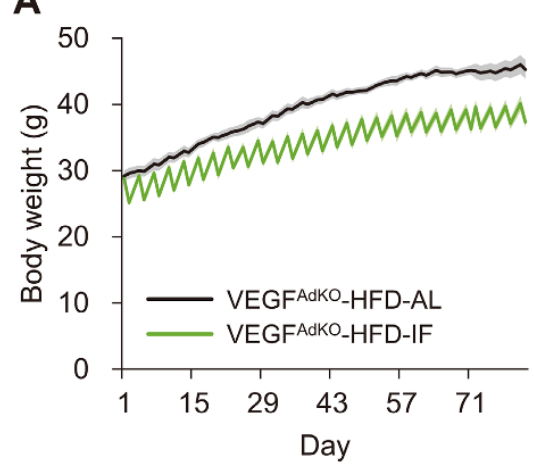

D

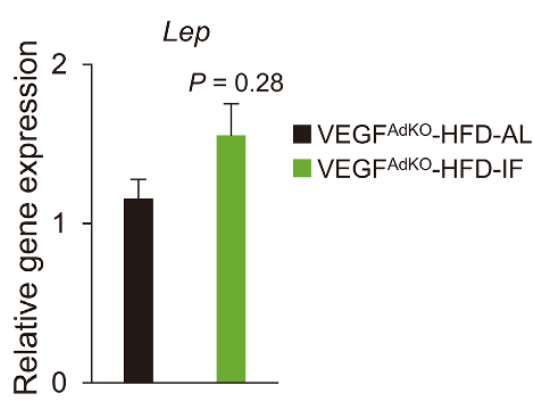

B 3 VEGFAKKO-HFD-AL

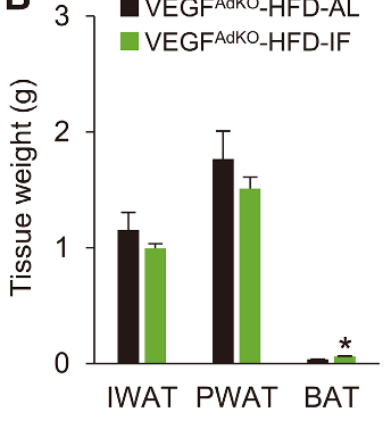

E

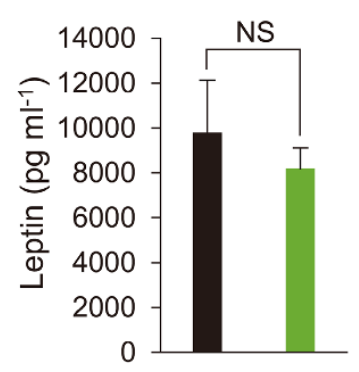

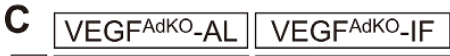

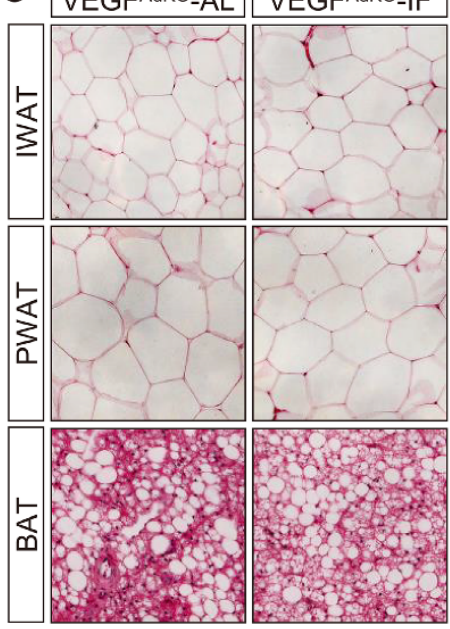

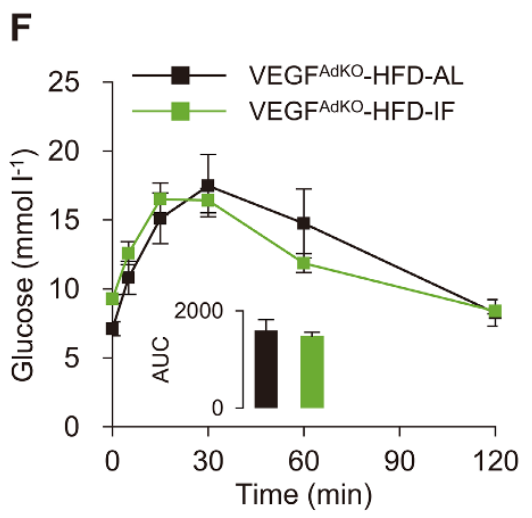
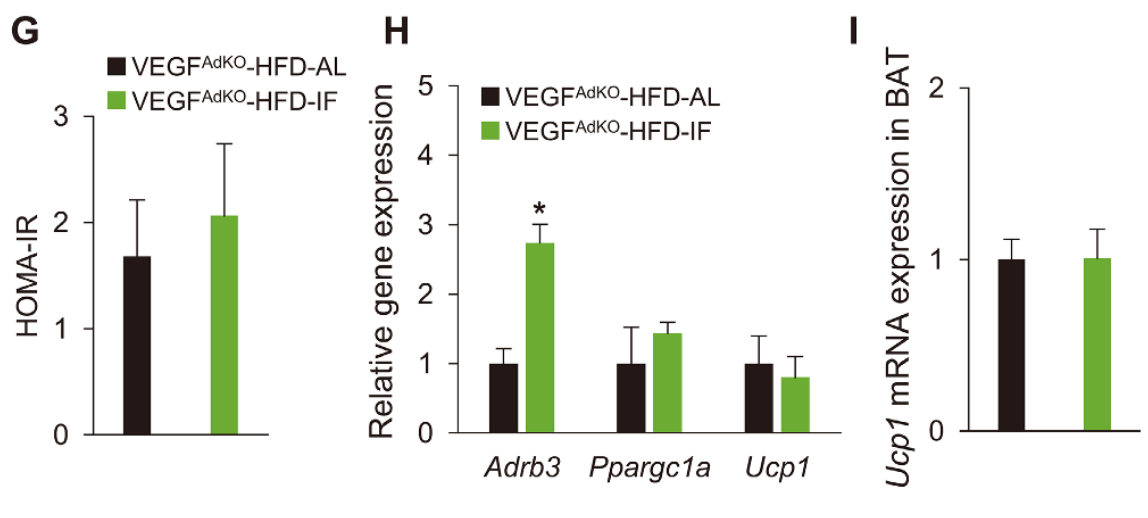

Figure 4 Adipose-VEGF is required for IF-mediated metabolic benefits. (A) Body weight measurements of aP2-Cre; Vegfa ${ }^{f l o x} / f l o x$ mice $\left(V\right.$ GFF $^{\text {AdKO }}$ ) subjected to AL and IF under HFD feeding (VEGF ${ }^{\text {AdKO }}$-HFD, AL/IF: $\left.n=5 / 6\right)$. (B) Tissue weight of IWAT, PWAT, and BAT in VEGF ${ }^{\text {AdKO }}$-HFD-AL and -IF mice. (C) H\&E-stained sections of IWAT, PWAT, and BAT show no noticeable differences between VEGF ${ }^{\text {AdKO }}$-HFD-AL and -IF mice. (D) Lep mRNA expression of PWAT in VEGF ${ }^{\text {AdKo }}$-HFD mice. (E) Plasma leptin levels. (F) GTT in VEGF ${ }^{\text {AdKO }}$-HFD mice. (G) HOMA-IR in VEGF ${ }^{\text {AdKO }}$-HFD mice. (H) Gene expression analysis revealed that IF increased sympathetic activation (Adrb3), but did not affect brown/beige adipocyte maker expression (i.e., Ucp1) in PWAT of VEGF ${ }^{\text {AdKO }}$ mice. (I) No changes in Ucp1 expression in BAT of VEGF ${ }^{\text {AdKO }}$ mice upon IF. Data are mean \pm SEM; two-tailed unpaired Student's $t$-test; * $P<0.05$ vs VEGF ${ }^{\text {AdKO }}$-HFD-AL. Lep, leptin.

illustrate the necessity and sufficiency of adipose-VEGF in IF-induced WAT browning and improved metabolism.

\section{Fasting and adipose-VEGF induce alternative activation of adipose macrophages}

Although VEGF is implicated in WAT browning in response to cold, exercise, and environmental enrichment
$[9,10]$, its underlying mechanism is not well understood. Recent studies have demonstrated that cold- and exercise-induced WAT browning is mediated through alternative activation of adipose macrophage by type 2 immune cytokines [41, 42]. In addition, both cold acclimation and exercise, similar to our fasting condition, also increase adipose-VEGF expression [10, 43]. Therefore, we spec- 
A

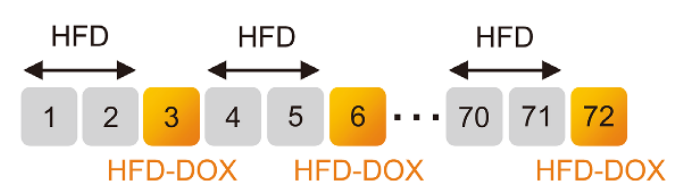

- VEGFCTRL : Control

- VEGFaP2-Tg : aP2-Cre;R26rtTA;Tet-O-VEGF164

VEGFAdipoq-Tg : Adipoq-Cre;R26 rTA;Tet-O-VEGF164
B

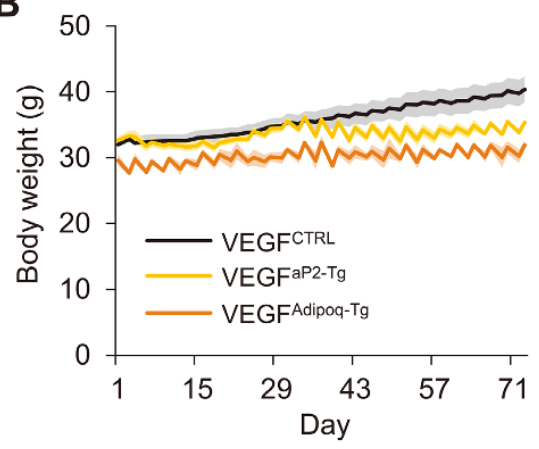

C

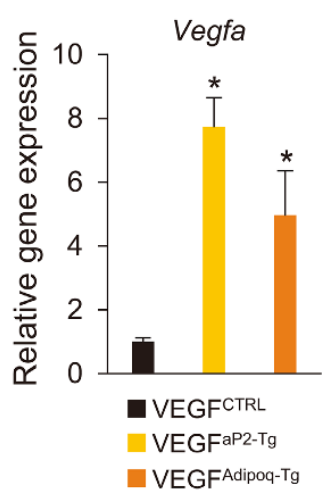

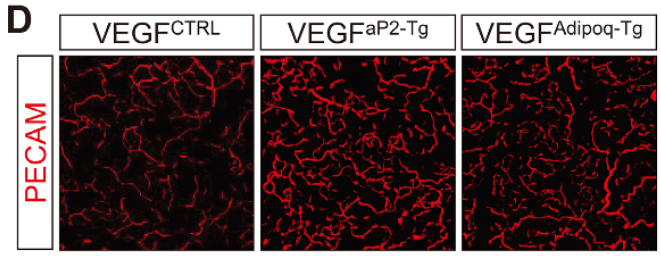
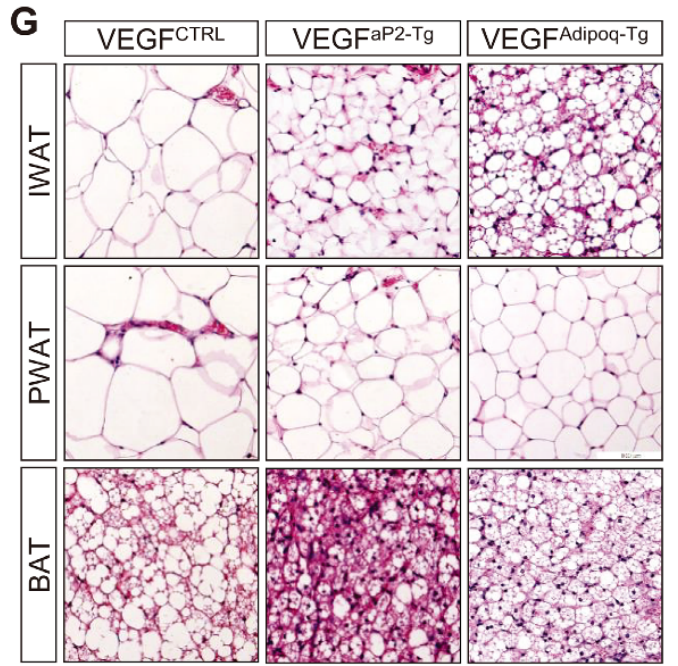
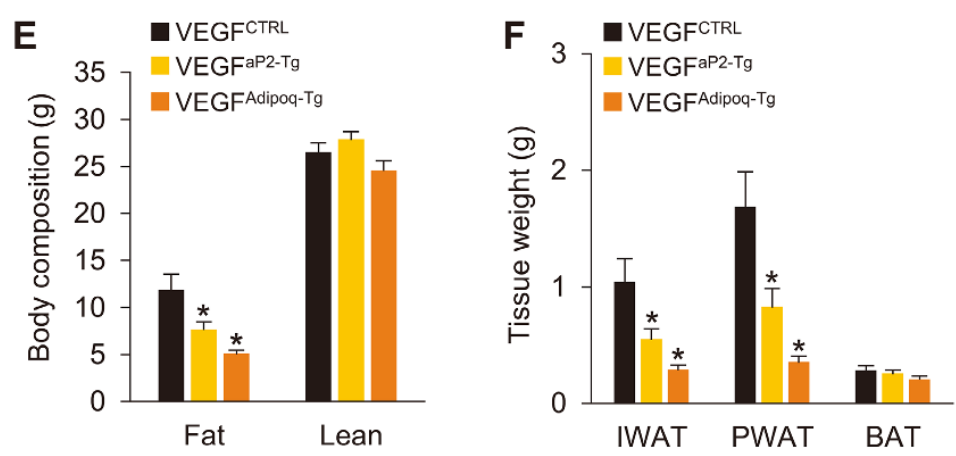
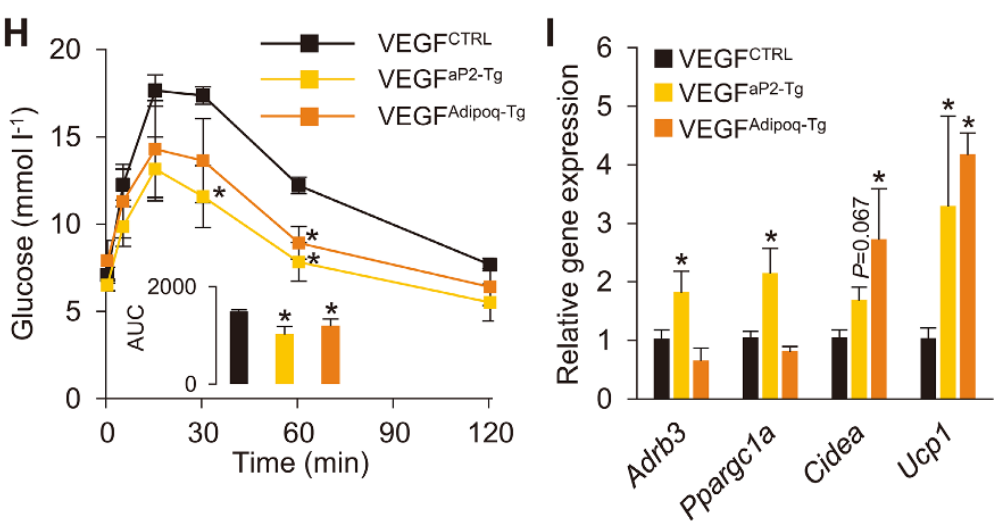

Figure 5 Intermittent adipose-VEGF overexpression is sufficient to mimic the IF-mediated metabolic benefits. (A) Schematic illustration of the 2:1 intermittent VEGF induction regimen and two different VEGF overexpression mouse models. (B) Body weight measurements during 10 weeks of adipose VEGF cyclic inductions. (C) Vegfa mRNA expression in PWAT at different fasting durations ( $n=5-6$ per group). (D) Representative microscopic images of PECAM-stained blood vessels in wholemount PWAT. (E) Body composition showing fat and lean mass. (F) Tissue weight of IWAT, PWAT, and BAT. (G) H\&E-stained sections of IWAT, PWAT, and BAT. (H) GTT. An insert graph shows AUC. (I) Gene expression analysis on WAT browning markers in PWAT. Data are expressed as mean \pm SEM $\left(\mathrm{VEGF}^{\mathrm{CTRL}}: n=10\right.$; VEGF ${ }^{\mathrm{aP2} 2 \mathrm{Tg}}: n=6$; and VEGF ${ }^{\text {Adipoq-Tg }}: n=5$ ); one- or two-way ANOVA with Student-Newman-Keuls post hoc analysis; ${ }^{*} P<0.05$ vs VEGF ${ }^{\text {CTRL }}$.

ulated that browning of WAT in IF might be mediated by alternatively activated macrophage. Indeed, we found that IF cycles led to alternative activation of adipose tissue macrophage indicated by elevated expression of M2 macrophage marker genes (e.g., Clec1Oa and Il10) in PWAT (Figure 6A), whereas expression of classical M1 macrophage marker genes was unchanged by IF. As a result, the M1/M2 gene expression ratio was reduced by IF (Supplementary information, Figure S10A). On the contrary, but consistent with the lack of WAT brown- 
A

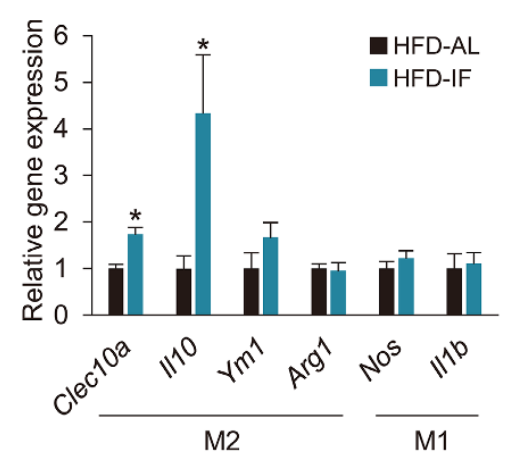

B

$\mathbf{E}$

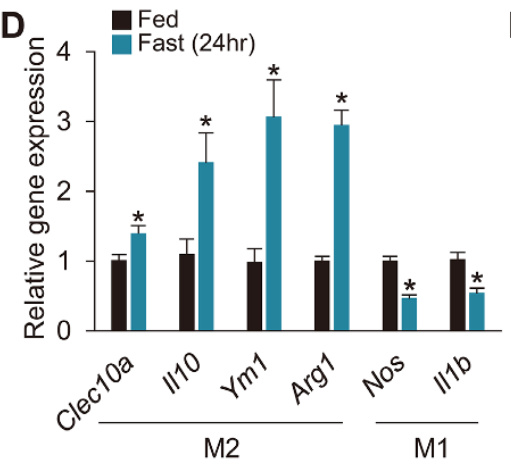

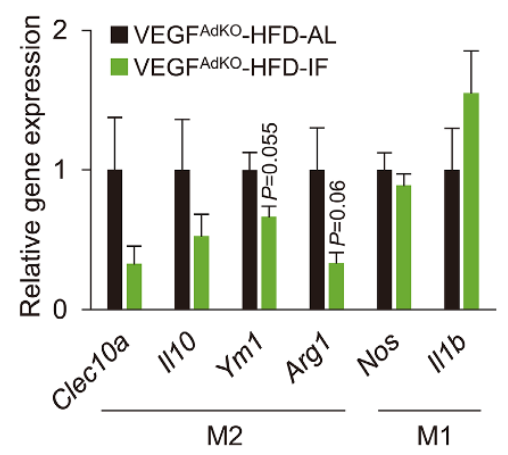

C

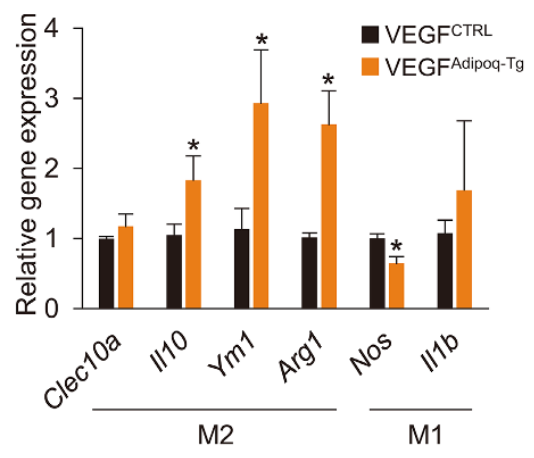

$\mathbf{F}$

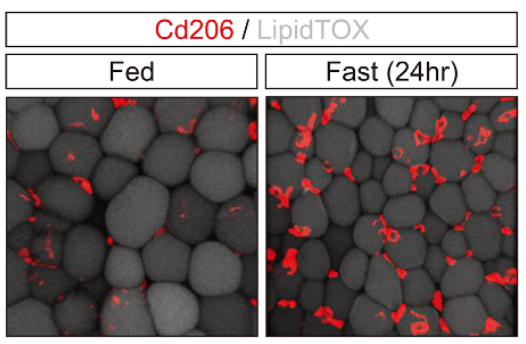

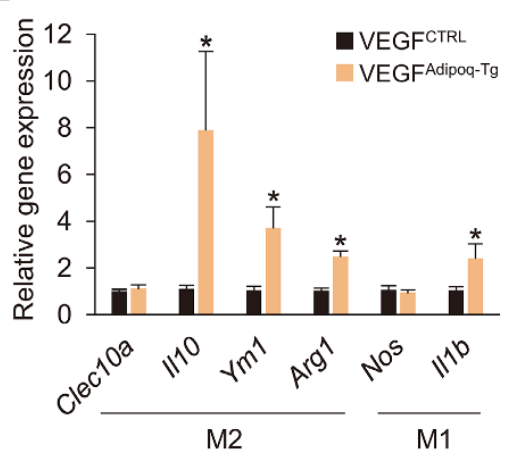

\section{G}
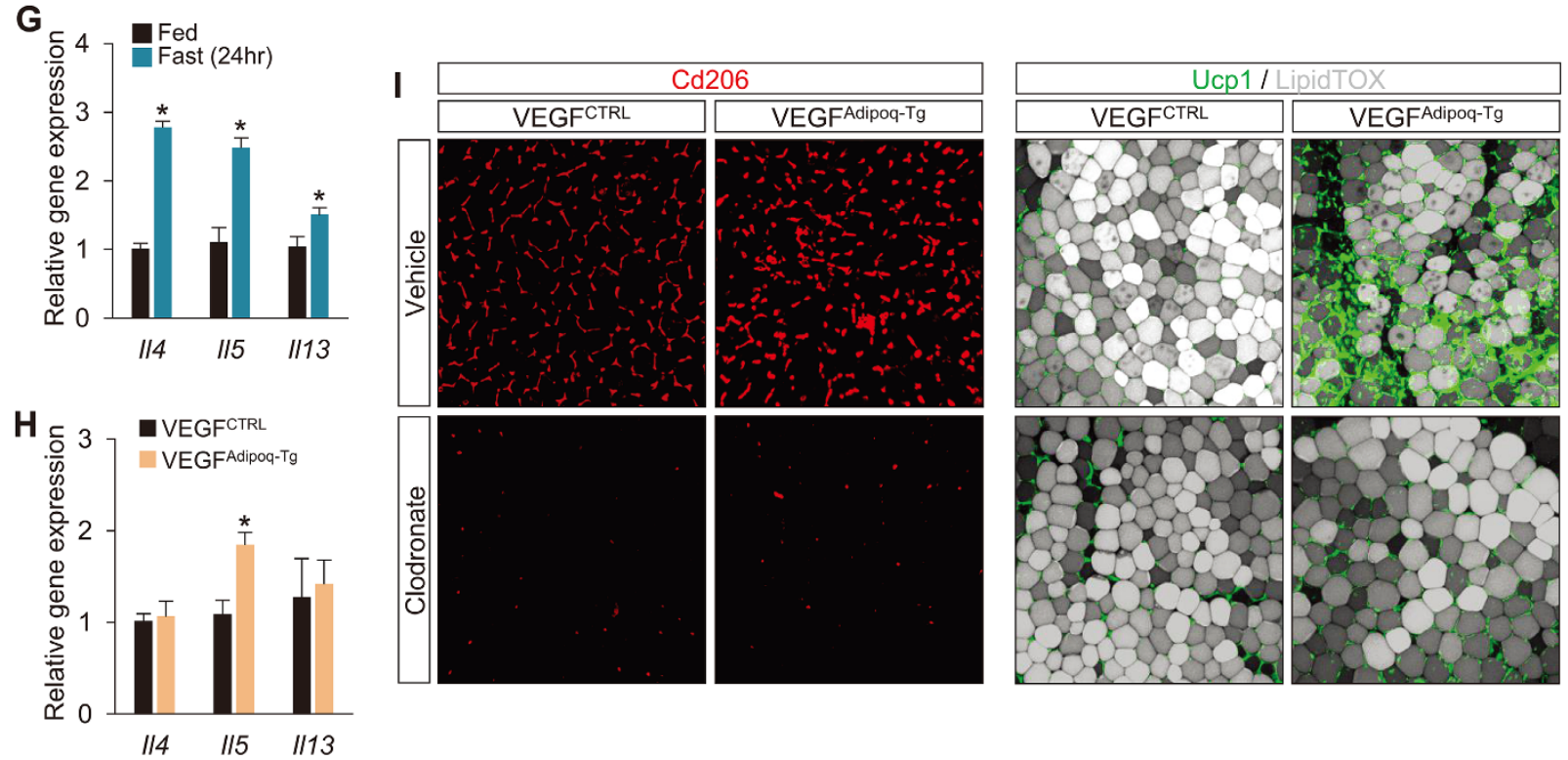

Figure 6 Fasting and adipose-VEGF induce alternative activation of macrophage. (A) M1/M2 macrophage marker gene expression analysis in HFD-AL and -IF mice. (B) M1/M2 macrophage marker gene expression in VEGF ${ }^{A d K O}$-HFD-AL and -IF mice. (C) M1/M2 macrophage marker gene expression after intermittent adipose-VEGF upregulation in VEGF ${ }^{\text {Adipoq-Tg }}$ mice. (D) M1/M2 macrophage marker gene expression analysis in fed and fasted (24 h) mice. (E) Representative images of M2 marker Cd206-stained cells in whole-mount PWAT of fed and fasted (24 h) mice. (F) M1/M2 macrophage marker gene expression analysis after acute adipose-VEGF upregulation in VEGF ${ }^{\text {Adipoq-Tg }}$ mice. (G) Type 2 cytokine gene expression in fed and fasted $(24 \mathrm{~h})$ mice. (H) Type 2 cytokine gene expression after acute adipose-VEGF upregulation (48 h) in VEGF ${ }^{\text {Adipoq-Tg }}$ mice. (I) Representative images of M2 macrophages and Ucp1 expression in WAT after acute adipose-VEGF upregulation in VEGF ${ }^{\text {Adipoq-Tg }}$ mice with treatments of vehicle or clodronate. Values are mean \pm SEM (post HFD-AL: $n=7$ and post HFD-IF: $n=9$ ); twotailed unpaired Student's $t$-test; ${ }^{*} P<0.05$ vs HFD-AL, VEGF ${ }^{\text {AdKO }}$-HFD-AL, VEGF ${ }^{\text {CRL }}$ or fed mice. See also Supplementary information, Figure $\mathrm{S} 7$. 
ing in VEGF ${ }^{\mathrm{AdKO}}$-HFD-IF mice, loss of adipose-VEGF completely eliminated the IF-mediated increase of alternatively activated adipose macrophage (Figure 6B; Supplementary information, Figure S10B and S10C). Similarly, the TN condition that blocked IF-stimulated WAT browning also hindered IF-induced M2 macrophage polarization (Supplementary information, Figure S10D and S10E). Notably, intermittent overexpression of adipose-VEGF using VEGF ${ }^{\text {Adipoq-Tg }}$ resulted in increased M2 marker gene expression, leading to a significant reduction in the M1/M2 ratio (Figure 6C; Supplementary information, Figure S10F and S10G). This finding was further verified by flow cytometry and whole-mount imaging, which showed that VEGF ${ }^{\text {Adipoq-Tg }}$ mice exhibited increased expression of $\mathrm{Cd} 206$ and $\mathrm{Cd} 301$ in WAT macrophage with a reduced M1/M2 ratio (i.e., $\mathrm{Cd} 11 \mathrm{c}^{+} /$ $\mathrm{Cd} 206^{+}$and $\mathrm{Cd} 11 \mathrm{c}^{+} / \mathrm{Cd} 301^{+}$), compared to $\mathrm{VEGF}^{\mathrm{CTRL}}$ mice (Supplementary information, Figure S10H-S10J). Collectively, these data demonstrate that IF and adipose-VEGF cycles promote alternative activation of adipose macrophage closely linked to WAT browning.

Importantly, even one-time 24-h fasting is sufficient to induce M2 macrophage polarization as evidenced by increased M2 macrophages and their marker gene expression (e.g., Clea10a, Il10, $\mathrm{Ym} 1$ and $\mathrm{Arg} 1$ ) as well as decreased expression of M1 marker genes (e.g., Nos and $I l l b$; Figure 6D and 6E; Supplementary information, Figure S11A). This suggests that these acute changes are repeated during IF, thereby facilitating its metabolic benefits. In addition, acute induction (i.e., $48 \mathrm{~h}$ ) of adipose $V e g f a$ overexpression $(\sim 3.5$-fold $)$ using VEGF $^{\text {Adipoq-Tg }}$ mice, whose level is comparable to $24-\mathrm{h}$ fasted mice (Figure 3C), is sufficient to increase M2 macrophage (Figure 6F; Supplementary information, Figure S11BS11E). However, direct administration of both mouse and human VEGF to Raw264.7 macrophages did not affect their properties, in contrast to interleukin-4 (IL-4) and lipopolysaccharide treatments that promote M2 and M1 polarizations, respectively (Supplementary information, Figure S11F). We found that a 24-h fasting upregulates gene expression of $\mathrm{Il}-4, \mathrm{Il}-5$ and $\mathrm{Il}-13$ (Figure 6G), type 2 cytokines important for the activation of M2 macrophage [5]. Acute induction of adipose VEGF also elevated IL-5 gene expression (Figure $6 \mathrm{H}$ ). These results suggest that VEGF-mediated M2 macrophage polarization is indirectly regulated, possibly via type 2 immune signaling.

Next, we tested whether adipose macrophages and their M2 polarization are required for VEGF-induced WAT browning. Consistent with a recent report [44], acute induction of adipose VEGF in both VEGF ${ }^{\text {Adipoq-Tg }}$ and $\mathrm{VEGF}^{\mathrm{a} 2 \mathrm{P}-\mathrm{Tg}}$ mice resulted in rapid browning of WAT with elevated Ucp1 expression (Figure 6I and Supple- mentary information, Figure S11G). However, this effect was markedly blocked by clodronate-induced macrophage depletion. Taken together, our results suggest that fasting-induced adipose-VEGF is a driver of M2 macrophage activation, which underlies IF-mediated adipose thermogenesis and associated metabolic benefits.

VEGF expression in human WAT correlates with M2 macrophage and WAT browning

To examine whether the adipose VEGF-M2 macrophage-WAT browning axis described above is relevant in humans, we utilized RNA-seq data of 350 human adipose tissues from the Genotype-Tissue Expression (GTEx) project [45] to calculate the correlation between VEGFA gene expression and expression levels of M2-associated genes, M1-associated genes, and beige adipocyte-associated genes [46-48] (Supplementary information, Table S2). The correlation heatmap using unbiased hierarchical clustering revealed that M2-associated genes positively correlated with VEGFA expression and were also clustered with beige adipocyte genes (Figure 7A). In contrast, most M1-associated genes negatively correlated with $V E G F A$ expression. For example, as shown in the scatter plot, expression of the M2 macrophage genes ILIRI and $A B H D 5$ [49], as well as the beige/brown adipocyte markers CIDEA and NDUFS2 [50], were positively correlated with $V E G F A$ expression, whereas negative correlation with $V E G F A$ was shown with the M1 macrophage genes $N R 3 C 2$ and ITGB7 [51] (Figure 7B). After applying the threshold of adjusted $P<0.05$ in VEGFA correlation with the gene set enrichment analysis (GSEA), we identified 24 out of 31 M2-associated genes displaying a positive correlation with VEGFA, 23 out of 30 beige adipocyte genes displaying a positive correlation, and 29 out of 32 M1-associated genes displaying a negative correlation (Figure 7C and Supplementary information, Figure S12). Together with our mouse data, these results suggest that the adipose-VEGF expression level, which is elevated by IF, is not only associated with vasculature, but is also a metabolic index indicating M2 macrophage activation and beige adipocyte development in both humans and mice (Figure 7D).

\section{Discussion}

Accumulating evidence suggests that IF provides various biological benefits in animal models and humans [13, 14]. For example, the 5:2 diet, which involves $C R$ for 2 non-consecutive days a week and unconstrained eating the other 5 days, has become a popular IF regimen and has a potential to be considered for medical interventions [13]. However, it remains elusive whether the effects 
A

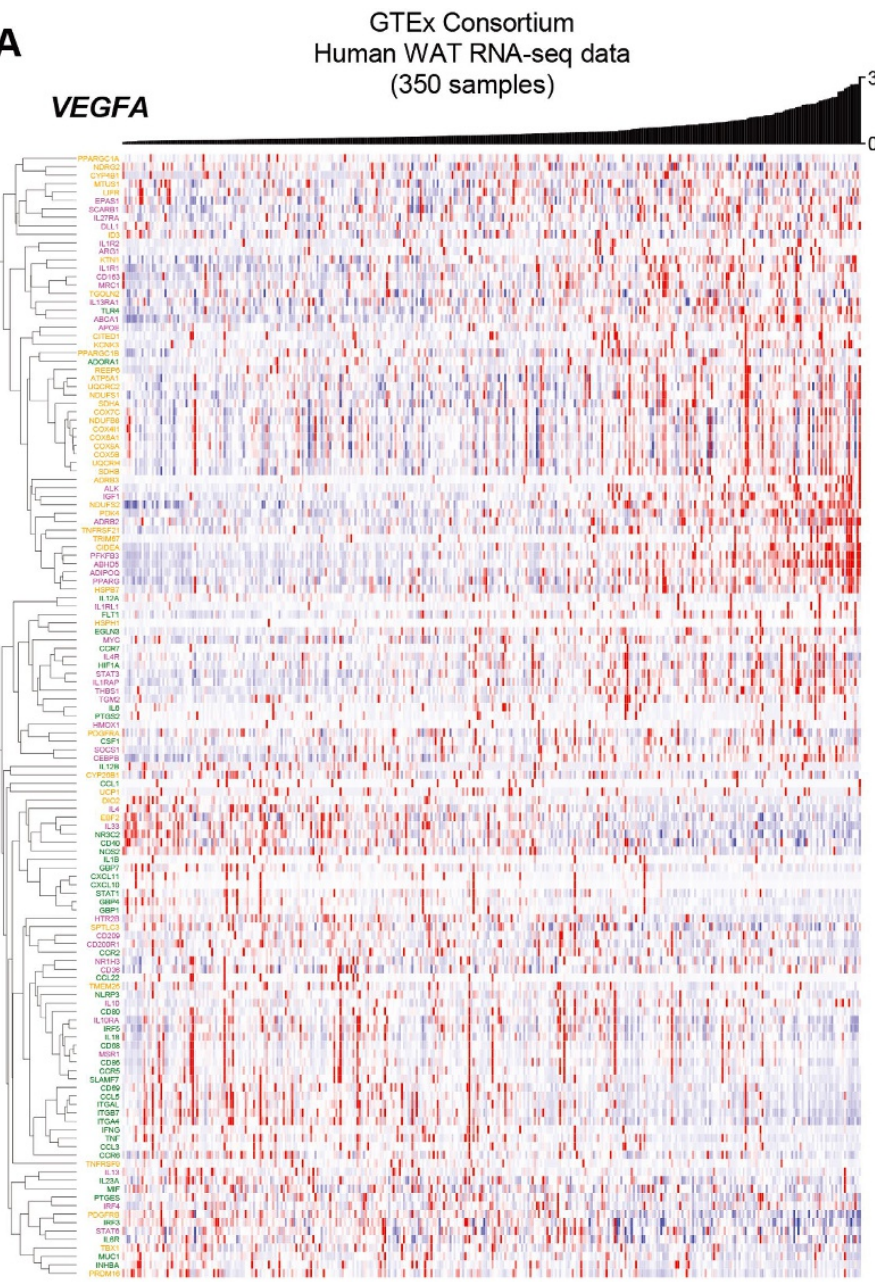

M2 macrophage gene Beige/brown adipocyte gene - M1 macrophage gene

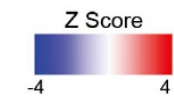

C

Number of genes correlated with VEGFA

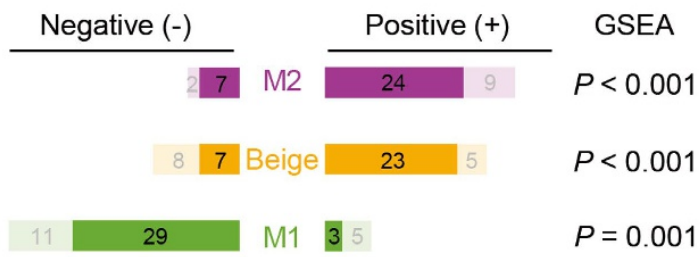

B
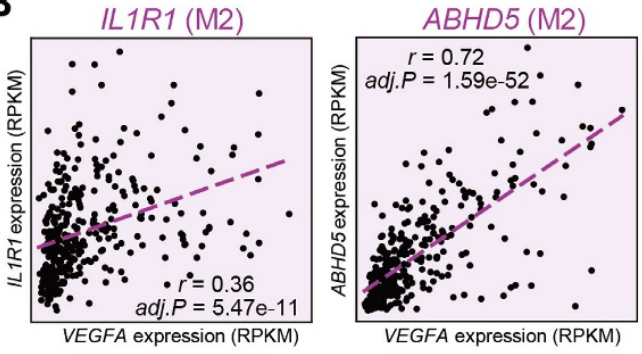

CIDEA (Beige)

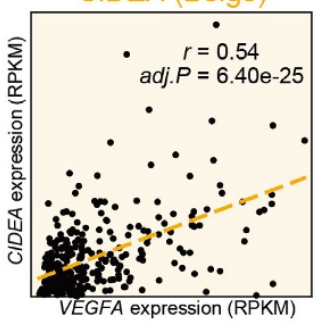

NDUFS2 (Beige)

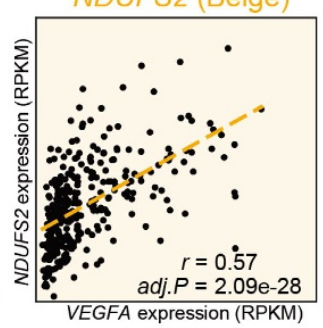

NR3C2 (M1)
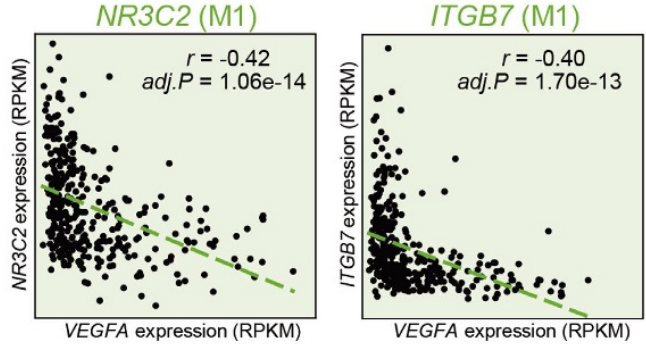

D

Intermittent Fasting
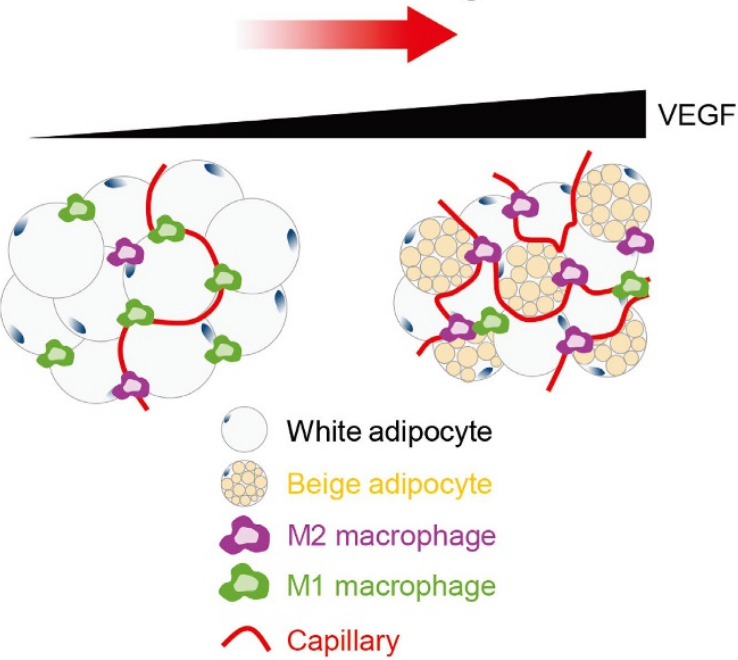

Figure 7 VEGF expression in human WAT correlates with M2 macrophage and WAT browning. (A) A correlation heatmap of VEGFA gene with unsupervised hierarchical clustering of M1/M2 macrophages- and beige/brown adipocyte-associated genes in human WAT. A histogram of VEGFA gene expression level (RPKM) is shown on the top of the heatmap. (B) Representative scatter plots showing correlation of VEGFA with IL1R1 \& ABHD5 (M2), CIDEA \& NUDFS2 (beige), and NR3C2 \& ITGB7 (M1) genes. (C) Summary of VEGFA correlation with M2, beige, and M1-associated genes. Permutation $P$-values with the GSEA are shown. (D) Schematic model of IF-mediated VEGF expression underlying adipose thermogenesis and M2 macrophage polarization. GSEA, gene set enrichment analysis. 
upon IF, often tested by alternate day fasting in rodent models [19], are attributed to reduced calorie intake and/ or modified eating pattern. The present study, using an isocaloric mouse model with a 2:1 IF regimen, demonstrates calorie-independent metabolic benefits of IF. Together with recent important findings on time-restricted feeding and a fasting-mimicking diet (FMD) [52, 53], our data support the significance of not only "what/how much' but also 'when/how often' in energy intake to sustain energy homeostasis and metabolic health. Our 2:1 IF rodent model provides an excellent platform for examining the mechanisms of isocaloric IF to prevent against various obesity-associated metabolic dysfunction.

Both IF and CR impact on nearly all metabolic organs/ tissues including hypothalamus, liver, skeletal muscle, and adipose tissues $[13,14]$. However, the key organ/ tissue driving the IF-induced metabolic benefits is not clearly defined. In the present study, our results posit that adipose tissues are not only an energy reservoir in fasting, but also play a pivotal role in IF-mediated metabolic benefits, particularly by regulating VEGF production. As sufficient vascularization is essential for adipose tissue development and function including cold-, exercise-, or environment-induced adaptive thermogenesis [9-11, $54,55]$, the activation of the adipose-VEGF pathway has been suggested as a new therapeutic strategy for the treatment of obesity and diabetes [56]. We demonstrate in this study that fasting is a physiological means of increasing adipose Vegfa gene expression (Figure 3 ), in addition to exercise [11] and cold exposure [10]. Accordingly, IF confers similar metabolic impacts as physical exercise and cold exposure (e.g., browning of WAT) with markedly increased adipose vasculature. Our adipose VEGF loss- and gain-of-function mouse models further illustrate that repetition of fasting-induced adipose-VEGF expression underlies IF-mediated metabolic benefits. Interestingly, elevated VEGF expression was mainly noted in IWAT (i.e., subcutaneous WAT) or BAT of mice subjected to cold exposure [10,57], whereas fasting-mediated augmentation of VEGF expression was greater in PWAT (i.e., visceral WAT) than in IWAT or BAT. Consistently, the profound weight loss in PWAT seen in our study (Supplementary information, Figure S1) and other study [52] indicates that visceral WAT is apparently more sensitive to energy restriction. Together, these observations suggest that, although systemic metabolic outcomes by elevated VEGF expression in different adipose depots with different physiological stimuli appear to be comparable (i.e., improved glucose handling with elevated energy expenditure), subcutaneous WAT, visceral WAT, or BAT have their distinctive physiological and adaptive metabolic properties. This further suggests that combinatory stimuli to activate both subcutaneous and visceral WAT, such as IF and exercise or IF and cold exposure, could generate synergistic metabolic benefits.

It is now well appreciated that adipose thermogenesis, particularly browning of WAT, improves whole-body metabolism in response to various external and internal stimuli [3], and that adipose-VEGF is clearly implicated in this process [6-9]. An improved supply of oxygen and nutrient via increased angiogenesis in adipose tissues can account for the necessity of adipose-VEGF in the adaptive responses to physiological stimuli and stress, but may not fully explain the sufficiency of adipose-VEGF in the induction of adipose thermogenesis as seen in several different transgenic mouse models. Of note, it appears that VEGF receptors, such as VEGFR1 (Flt1) and VEGFR2 (Flk1), are not expressed in preadipocytes or mature adipocytes [6], leading us to postulate that VEGF acts indirectly in adipose tissue remodeling. Indeed, a recent study has demonstrated that adipose-VEGF induces endothelial PDGF-CC in response to $\beta 3$-adrenergic activation, thereby stimulating PDGFR $\alpha^{+}$-progenitor beige adipocytes in WAT [58]. However, we did not find substantial changes of $P d g f c$ expression in the WAT of fasted or VEGF ${ }^{\text {Adipoq-Tg }}$ mice (data not shown). Importantly, our data and a recent study [59] demonstrated that lifestyle interventions, IF, and CR triggered alternative activation (M2) of adipose macrophage, and the type 2 innate immune response that plays a critical role in this adipose thermogenic remodeling [5]. These immunological changes were also observed in mice with periodic overexpression of adipose-VEGF (driven by Adipoq-Cre and $a P 2-C r e$ ), similar to a previous study [8], but not in the mice lacking IF-mediated WAT browning due to thermoneutrality (TN-HFD-IF) or loss of adipose-VEGF (VEGF ${ }^{\text {AdKO }}$ ). In addition, both a one-time 24-h fasting and acute adipose-VEGF overexpression without fasting induce alternative activation of adipose macrophage, whereas depletion of macrophage in the presence of adipose-VEGF overexpression abrogated the WAT browning. Together, our results demonstrate that (1) M2 macrophage polarization in IF mice is not simply a consequence of leanness, but a driving force of adipose thermogenesis and metabolic benefits, and (2) adipose-VEGF plays a role in the regulation of M2 macrophage activation as a rapid adaptive response to energy restriction, likely even prior to angiogenesis. Furthermore, (3) the M1/M2 macrophage balance, rather than the number of macrophage, may be critical for adipose thermogenesis, as all fasted mice had reductions in the number of adipose macrophage regardless of conditions.

Nevertheless, little is known about the molecular mechanism underlying fasting- or adipose-VEGF-me- 
diated M2 polarization. Our study showed that M2 macrophage polarization was indirectly regulated by VEGF, similar to the previous study [8]. What could be the mechanism of this VEGF-mediated M2 transition? Among various upstream signaling pathways implicated in M2 polarization [60], we found elevated type2 cytokines by both fasting (IL-4, IL-5 and IL-13) and acute adipose-VEGF induction (IL-5), suggesting that adipose-VEGF may act on other upstream immune cells, such as eosinophils, T-helper cell 2 (Th2), or type 2 innate lymphoid cells (ILC2), to promote M2 polarization [61]. Therefore, future studies delineating a precise role (e.g., activation or recruitment of immune cells) and its molecular mechanism (e.g., through VEGFR1, VEGFR2, and VEGFB [56]) of VEGF signaling in adipose immunometabolism should uncover the fundamental mechanism in WAT browning. Furthermore, other adipose factors that we observed to be affected by IF, such as leptin, neuregulin 4, adiponectin and adipsin, could play a role in fasting-mediated immunometabolic responses. This might be particularly expected for leptin, whose expression level is reduced by IF or fasting in WAT and BAT (not in skeletal muscle or liver). Leptin modulates a wide range of immune and inflammatory processes, and leptin-deficient mast cells undergo M2 polarization [62]. Given that leptin could synergistically act with VEGF (e.g., in angiogenesis) [63], it will be of interest to examine the implication of leptin in fasting- and VEGF-mediated metabolic benefits. On the other hand, these adipose factors may be involved in the non-thermogenic effects of IF. Although thermoneutrality markedly reduced the metabolic benefits of IF with inhibition of WAT browning, it did not completely eliminate the IF's effects as adipose-derived factors showed similar gene expression (data not shown). As previous studies demonstrate that neuregulin 4 and adipsin are implicated in inhibition of hepatic steatosis [32] and improvement of insulin secretion [64], respectively, the non-thermogenic benefits of IF may be attributed to these adipose-derived factors or possibly other physiological responses, such as reduced adipose inflammation and increased insulin sensitivity (Figure 2A, Cluster \#3).

IF has been practiced in human clinical settings with various fasting regimens, and most studies revealed similar beneficial health effects such as body weight reduction and increased insulin sensitivity $[18,21,65$, 66]. However, IF can be stressful to body and mind, because 24 -h fasting can reduce body weight by $2 \%-3 \%$ in humans [65] and 5\%-10\% in mice. Although it is not easy to compare the effects of fasting in mice directly with humans due to differences in basal metabolic rate, food intake, as well as age differences [67], IF appears to improve eating behavior and mood in humans [68]. It will be important to overcome potential limitations by developing a method to improve the practicality of IF if our findings are to have translational perspective. For example, a method like FMD that provides various metabolic benefits in both mice and humans $[18,21,66]$ would improve the practicality of IF in a clinical setting. Further rigorous studies are required to examine: (1) whether the beneficial effects of IF last after stopping the fasting (i.e., if there is a rebound effect); (2) whether there is any potential harm of IF; and (3) whether IF benefits apply to all generations (i.e., an age-dependent issue) and disease conditions. Notably, 5 weeks of IF was able to drive beneficial metabolic effects in 8-monthold mice with HFD-induced obesity (unpublished data), further emphasizing the therapeutic potential of IF in age-associated metabolic abnormalities. To further determine the mechanism of IF, it is necessary to test whether fasting (or IF) induces VEGF expression in human adipose tissues, and whether fasting-induced adipose VEGF promotes M2 polarization and WAT browning in humans. Thus, future studies are warranted to examine the adaptive response of human adipose tissues to fasting or IF intervention via thermogenic and even non-thermogenic pathways, in association with the proposed mechanisms such as circadian rhythm $[53,69]$, gut microbiome changes [70], and sleep regulation.

In summary, our data demonstrate that metabolic benefits of IF are largely mediated by adipose thermogenesis without overall caloric reduction. We showed that fasting-induced adipose-VEGF plays a key role in WAT browning through M2 macrophage activation. This finding is further supported by human adipose tissue gene expression analysis illustrating positive correlations between adipose-VEGF expression and both M2 macrophage and WAT browning. Together, the present study illustrates the role of IF-induced adipose-VEGF in remodeling the immunometabolic property of adipose tissue, highlighting the importance of eating pattern and physiological fasting duration to sustain metabolic homeostasis.

\section{Materials and Methods}

\footnotetext{
Animals

All animal experimental protocols approved by the Animal Care Committee of the Centre of Phenogenomics conformed to the standards of the Canadian Council on Animal Care. No statistical methods were used to predetermine sample size. The investigators were not blinded to allocation during experiments. The colony was housed in a specific pathogen-free (SPF) facility in ventilated cages with controlled environment settings $\left(21-22^{\circ} \mathrm{C}, 30 \%-60 \%\right.$ humidity for normal housing), 12-h light/dark cycles, and free access
} 
to water. For the thermoneutrality experiment, mice were housed at $30{ }^{\circ} \mathrm{C}$. C57BL/6J mice were purchased from the Jackson Laboratory. aP2-Cre, Adipoq-Cre, Ucp1-Cre, Vegfa ${ }^{\text {floxflox }}$; TetO-Vegf164, $\operatorname{Rosa}_{2} 6^{r T A}$ and Rosa2 $6^{m T / m G}$ mice were described previously [6, 71, 72]. Age-matched littermate controls were used for each experiment.

\section{IF regimen and diets}

Body weight-matched 8-week-old male C57BL/6J, VEGF ${ }^{\text {AdKo }}$, and Ucp 1-Cre;Rosa $26^{m T / m G}$ mice were randomly divided into two groups: AL and IF groups. Mice were fed either ND (Harlan \#2918; 17\% fat) or 45\% HFD (\#D12451, Research Diets). Mice in the IF group were subjected to the $2: 1$ IF regimen, comprising 1 day of fasting followed by 2 days of feeding; the food was removed at 12:00 PM and then provided again the following day (24 h later) at 12:00 PM. Mice in the AL group were handled equivalently. To induce periodic VEGF overexpression in adipose tissues, VEG$\mathrm{F}^{\mathrm{CTRL}}, \mathrm{VEGF}^{\mathrm{aP2}-\mathrm{Tg}}$, and $\mathrm{VEGF}^{\mathrm{Adipoq}-\mathrm{Tg}}$ mice were subjected to $45 \%$ HFD-DOX (Research Diets) once every 3 days (Figure 5A).

\section{Statistical analysis}

All results are presented as mean \pm SEM. Statistical significance of differences among groups was determined by two-tailed unpaired and paired Student's $t$-test as well as analysis of variance (ANOVA) with post hoc analysis, Student-Newman-Keuls, using Sigma Stat (SPSS) or PRISM 5.0 (GraphPad). Differences with $P$ $<0.05$ were considered statistically significant.

Other methods are described in Supplementary information, Data S1.

\section{Acknowledgments}

We thank Drs Daniel Drucker, Tony Lam, Warren Lee, and So-Young Park for critical reading of the manuscript; Drs Javier Jaldin-Fincati, Azadeh Yeganeh, and Hisato Konoeda for experimental support and advice; and Jannel Liu for animal husbandry. We also thank Dr Jason Fung for scientific discussion and Elizabeth Lebedev for illustration. This work was funded by grants from the Natural Sciences and Engineering Research Council (NSERC) of Canada, Pilot and Feasibility Study Grant of Banting \& Best Diabetes Centre (BBDC), Centre for Healthy Active Kids (CHAK) Micro-grant and Sickkids Start-up fund to H-KS, the Canadian Institutes of Health Research (CIHR) to C-CH, and Medical Research Center Program (2015R1A5A2009124) through National Research Foundation of Korea (NRF) funded by Ministry of Science, ICT, and Future Planning to J-RK. K-HK and JHM are supported by the Restracomp fellowship from The Hospital for Sick Children. JES is supported by a fellowship from the Canadian Diabetes Association. JHL is supported by the Restracomp scholarship from The Hospital for Sick Children.

\section{Author Contributions}

$\mathrm{K}-\mathrm{HK}, \mathrm{C}-\mathrm{CH}$, and $\mathrm{H}-\mathrm{KS}$ conceived, designed, and supervised the research project. K-HK, YHK, JES, JHL, JHM, SK, MSC, $\mathrm{K}-\mathrm{OD}$ and J-AY performed mouse metabolic experiments. K-HK and YHK analyzed mouse metabolic data. K-HK, YHK, and JHL performed gene expression analysis with assistance from SK and MSC. Histology was conducted by JZ, and analyzed by K-HK, YHK, KF, and FL. JES performed whole-mount adipose tissue staining for blood vessel and macrophage. JGP and SMIH analyzed mouse RNA-seq data. JGP and K-HK performed human gene correlation analysis. AN, AK, and J-RK provided scientific discussion and technical support. K-HK, C-CH, and H-KS wrote the manuscript. All authors discussed the results and commented on the manuscript.

\section{Competing Financial Interests}

The authors declare no competing financial interests.

\section{References}

1 Rutkowski JM, Stern JH, Scherer PE. The cell biology of fat expansion. J Cell Biol 2015; 208:501-512.

2 Stern JH, Scherer PE. Adipose tissue biology in 2014: advances in our understanding of adipose tissue homeostasis. Nat Rev Endocrinol 2015; 11:71-72.

3 Kajimura S, Spiegelman BM, Seale P. Brown and beige fat: physiological roles beyond heat generation. Cell Metab 2015; 22:546-559.

4 Wang W, Seale P. Control of brown and beige fat development. Nat Rev Mol Cell Biol 2016; 17:691-702.

5 Odegaard JI, Chawla A. Type 2 responses at the interface between immunity and fat metabolism. Curr Opin Immunol 2015; 36:67-72.

6 Sung HK, Doh KO, Son JE, et al. Adipose vascular endothelial growth factor regulates metabolic homeostasis through angiogenesis. Cell Metab 2013; 17:61-72.

7 Sun K, Wernstedt Asterholm I, Kusminski CM, et al. Dichotomous effects of VEGF-A on adipose tissue dysfunction. Proc Natl Acad Sci USA 2012; 109:5874-5879.

8 Elias I, Franckhauser S, Ferre T, et al. Adipose tissue overexpression of vascular endothelial growth factor protects against diet-induced obesity and insulin resistance. Diabetes 2012; 61:1801-1813.

9 During MJ, Liu X, Huang W, et al. Adipose VEGF links the white-to-brown fat switch with environmental, genetic, and pharmacological stimuli in male mice. Endocrinology 2015; 156:2059-2073.

10 Xue Y, Petrovic N, Cao R, et al. Hypoxia-independent angiogenesis in adipose tissues during cold acclimation. Cell Metab 2009; 9:99-109.

11 Stanford KI, Middelbeek RJ, Townsend KL, et al. A novel role for subcutaneous adipose tissue in exercise-induced improvements in glucose homeostasis. Diabetes 2015; 64:20022014.

12 Gill S, Panda S. A smartphone app reveals erratic diurnal eating patterns in humans that can be modulated for health benefits. Cell Metab 2015; 22:789-798.

13 Longo VD, Panda S. Fasting, circadian rhythms, and time-restricted feeding in healthy lifespan. Cell Metab 2016; 23:1048-1059.

14 Longo VD, Mattson MP. Fasting: molecular mechanisms and clinical applications. Cell Metab 2014; 19:181-192.

15 Anson RM, Guo Z, de Cabo R, et al. Intermittent fasting dissociates beneficial effects of dietary restriction on glucose metabolism and neuronal resistance to injury from calorie intake. Proc Natl Acad Sci USA 2003; 100:6216-6220.

16 Fontana L, Partridge L. Promoting health and longevity 
through diet: from model organisms to humans. Cell 2015; 161:106-118.

17 Harvie MN, Pegington M, Mattson MP, et al. The effects of intermittent or continuous energy restriction on weight loss and metabolic disease risk markers: a randomized trial in young overweight women. Int J Obes 2011; 35:714-727.

18 Patterson RE, Laughlin GA, LaCroix AZ, et al. Intermittent fasting and human metabolic health. J Acad Nutr Diet 2015; 115:1203-1212.

19 Boutant M, Kulkarni SS, Joffraud M, et al. SIRT1 gain of function does not mimic or enhance the adaptations to intermittent fasting. Cell Rep 2016; 14:2068-2075.

20 Harvie M, Wright C, Pegington M, et al. The effect of intermittent energy and carbohydrate restriction v. daily energy restriction on weight loss and metabolic disease risk markers in overweight women. Br J Nutr 2013; 110:1534-1547.

21 Varady KA, Bhutani S, Klempel MC, et al. Alternate day fasting for weight loss in normal weight and overweight subjects: a randomized controlled trial. Nutr J 2013; 12:146.

22 Martin A, Normand S, Sothier M, Peyrat J, Louche-Pelissier C, Laville M. Is advice for breakfast consumption justified? Results from a short-term dietary and metabolic experiment in young healthy men. Br J Nutr 2000; 84:337-344.

23 Wan R, Camandola S, Mattson MP. Intermittent food deprivation improves cardiovascular and neuroendocrine responses to stress in rats. $J$ Nutr $2003 ; \mathbf{1 3 3}: 1921-1929$.

24 Gotthardt JD, Verpeut JL, Yeomans BL, et al. Intermittent fasting promotes fat loss with lean mass retention, increased hypothalamic norepinephrine content, and increased neuropeptide Y gene expression in diet-induced obese male mice. Endocrinology 2016; 157:679-691.

25 Bapat SP, Myoung Suh J, Fang S, et al. Depletion of fat-resident Treg cells prevents age-associated insulin resistance. Nature 2015; 528:137-141.

26 Rajakumari $\mathrm{S}, \mathrm{Wu} \mathrm{J}$, Ishibashi J, et al. EBF2 determines and maintains brown adipocyte identity. Cell Metab 2013; 17:562574.

27 Barquissau V, Beuzelin D, Pisani DF, et al. White-to-brite conversion in human adipocytes promotes metabolic reprogramming towards fatty acid anabolic and catabolic pathways. Mol Metab 2016; 5:352-365.

28 Mottillo EP, Balasubramanian P, Lee YH, Weng C, Kershaw EE, Granneman JG. Coupling of lipolysis and de novo lipogenesis in brown, beige, and white adipose tissues during chronic 33 -adrenergic receptor activation. J Lipid Res 2014; 55:2276-2286.

29 Cui X, Nguyen NL, Zarebidaki E, et al. Thermoneutrality decreases thermogenic program and promotes adiposity in highfat diet-fed mice. Physiol Rep 2016; 4.

30 Lehr S, Hartwig S, Sell H. Adipokines: a treasure trove for the discovery of biomarkers for metabolic disorders. Proteomics Clin Appl 2012; 6:91-101.

31 Deng Y, Scherer PE. Adipokines as novel biomarkers and regulators of the metabolic syndrome. Ann N Y Acad Sci 2010; 1212:E1-E19.

32 Wang GX, Zhao XY, Meng ZX, et al. The brown fat-enriched secreted factor Nrg4 preserves metabolic homeostasis through attenuation of hepatic lipogenesis. Nat Med 2014; 20:14361443.
33 Dodd GT, Decherf S, Loh K, et al. Leptin and insulin act on POMC neurons to promote the browning of white fat. Cell 2015; 160:88-104.

34 Tonello C, Giordano A, Cozzi V, et al. Role of sympathetic activity in controlling the expression of vascular endothelial growth factor in brown fat cells of lean and genetically obese rats. FEBS Lett 1999; 442:167-172.

35 Buzelle SL, MacPherson RE, Peppler WT, Castellani L, Wright DC. The contribution of IL-6 to $\beta 3$ adrenergic receptor mediated adipose tissue remodeling. Physiol Rep 2015; 3.

36 Granneman JG, Lahners KN, Chaudhry A. Molecular cloning and expression of the rat beta 3-adrenergic receptor. $\mathrm{Mol}$ Pharmacol 1991; 40:895-899.

37 Cho $\mathrm{CH}$, Koh YJ, Han J, et al. Angiogenic role of LYVE-1-positive macrophages in adipose tissue. Circ Res 2007; 100:e47-e57.

38 Li YL, Zhao H, Ren XB. Relationship of VEGF/VEGFR with immune and cancer cells: staggering or forward? Cancer Biol Med 2016; 13:206-214.

39 Pang C, Gao Z, Yin J, Zhang J, Jia W, Ye J. Macrophage infiltration into adipose tissue may promote angiogenesis for adipose tissue remodeling in obesity. Am J Physiol Endocrinol Metab 2008; 295:E313-E322.

40 Jeffery E, Berry R, Church CD, et al. Characterization of Cre recombinase models for the study of adipose tissue. Adipocyte 2014; 3:206-211.

41 Nguyen KD, Qiu Y, Cui X, et al. Alternatively activated macrophages produce catecholamines to sustain adaptive thermogenesis. Nature 2011; 480:104-108.

42 Rao RR, Long JZ, White JP, et al. Meteorin-like is a hormone that regulates immune-adipose interactions to increase beige fat thermogenesis. Cell 2014; 157:1279-1291.

43 Disanzo BL, You T. Effects of exercise training on indicators of adipose tissue angiogenesis and hypoxia in obese rats. $\mathrm{Me}$ tabolism 2014; 63:452-455.

44 Park J, Kim M, Sun K, An YA, Gu X, Scherer PE. VEGF-A-expressing adipose tissue shows rapid beiging and enhanced survival after transplantation and confers IL-4-independent metabolic improvements. Diabetes 2017; 66:1479-1490.

45 Consortium GT. Human genomics. the Genotype-Tissue Expression (GTEx) pilot analysis: multitissue gene regulation in humans. Science 2015; 348:648-660.

46 Shinoda K, Luijten IH, Hasegawa Y, et al. Genetic and functional characterization of clonally derived adult human brown adipocytes. Nat Med 2015; 21:389-394.

47 Roszer T. Understanding the mysterious M2 macrophage through activation markers and effector mechanisms. Mediators Inflamm 2015; 2015:816460.

48 Martinez FO, Gordon S. The M1 and M2 paradigm of macrophage activation: time for reassessment. F1000Prime Rep 2014; 6:13.

49 Natsuaki Y, Egawa G, Nakamizo S, et al. Perivascular leukocyte clusters are essential for efficient activation of effector $\mathrm{T}$ cells in the skin. Nat Immunol 2014; 15:1064-1069.

50 Sidossis L, Kajimura S. Brown and beige fat in humans: thermogenic adipocytes that control energy and glucose homeostasis. J Clin Invest 2015; 125:478-486.

51 Mauer J, Chaurasia B, Goldau J, et al. Signaling by IL-6 promotes alternative activation of macrophages to limit en- 
dotoxemia and obesity-associated resistance to insulin. Nat Immunol 2014; 15:423-430.

52 Brandhorst S, Choi IY, Wei M, et al. A periodic diet that mimics fasting promotes multi-system regeneration, enhanced cognitive performance, and healthspan. Cell Metab 2015; 22:86-99.

53 Hatori M, Vollmers C, Zarrinpar A, et al. Time-restricted feeding without reducing caloric intake prevents metabolic diseases in mice fed a high-fat diet. Cell Metab 2012; 15:848860.

54 Cao Y. Angiogenesis and vascular functions in modulation of obesity, adipose metabolism, and insulin sensitivity. Cell $\mathrm{Me}$ tab 2013; 18:478-489.

55 Shimizu I, Aprahamian T, Kikuchi R, et al. Vascular rarefaction mediates whitening of brown fat in obesity. J Clin Invest 2014; 124:2099-2112.

56 Robciuc MR, Kivela R, Williams IM, et al. VEGFB/VEGFR1-induced expansion of adipose vasculature counteracts obesity and related metabolic complications. Cell Metab 2016; 23:712-724.

57 Fredriksson JM, Nikami H, Nedergaard J. Cold-induced expression of the VEGF gene in brown adipose tissue is independent of thermogenic oxygen consumption. FEBS Lett 2005; 579:5680-5684.

58 Seki T, Hosaka K, Lim S, et al. Endothelial PDGF-CC regulates angiogenesis-dependent thermogenesis in beige fat. Nat Commun 2016; 7:12152.

59 Fabbiano S, Suarez-Zamorano N, Rigo D, et al. Caloric restriction leads to browning of white adipose tissue through type 2 immune signaling. Cell Metab 2016; 24:434-446.

60 Man K, Kutyavin VI, Chawla A. Tissue immunometabolism: development, physiology, and pathobiology. Cell Metab 2017; 25:11-26.

61 Molofsky AB, Nussbaum JC, Liang HE, et al. Innate lymphoid type 2 cells sustain visceral adipose tissue eosinophils and alternatively activated macrophages. J Exp Med 2013; 210:535-549.

62 Zhou Y, Yu X, Chen H, et al. Leptin deficiency shifts mast cells toward anti-inflammatory actions and protects mice from obesity and diabetes by polarizing M2 macrophages. Cell Metab 2015; 22:1045-1058.

63 Cao R, Brakenhielm E, Wahlestedt C, Thyberg J, Cao Y. Leptin induces vascular permeability and synergistically stimulates angiogenesis with FGF-2 and VEGF. Proc Natl Acad Sci USA 2001; 98:6390-6395.
64 Lo JC, Ljubicic S, Leibiger B, et al. Adipsin is an adipokine that improves $\beta$ cell function in diabetes. Cell 2014; 158:4153.

65 Heilbronn LK, Smith SR, Martin CK, Anton SD, Ravussin E. Alternate-day fasting in nonobese subjects: effects on body weight, body composition, and energy metabolism. Am J Clin Nutr 2005; 81:69-73.

66 Wei M, Brandhorst S, Shelehchi M, et al. Fasting-mimicking diet and markers/risk factors for aging, diabetes, cancer, and cardiovascular disease. Sci Transl Med 2017; 9.

67 Ellacott KL, Morton GJ, Woods SC, Tso P, Schwartz MW. Assessment of feeding behavior in laboratory mice. Cell $\mathrm{Me}$ tab 2010; 12:10-17.

68 Harvie M, Howell A. Potential benefits and harms of intermittent energy restriction and intermittent fasting amongst obese, overweight and normal weight subjects - a narrative review of human and animal evidence. Behav Sci 2017; 7.

69 LeCheminant JD, Christenson E, Bailey BW, Tucker LA. Restricting night-time eating reduces daily energy intake in healthy young men: a short-term cross-over study. Br J Nutr 2013; 110:2108-2113.

70 Thaiss CA, Zeevi D, Levy M, et al. Transkingdom control of microbiota diurnal oscillations promotes metabolic homeostasis. Cell 2014; 159:514-529.

71 Kong X, Banks A, Liu T, et al. IRF4 is a key thermogenic transcriptional partner of PGC-1alpha. Cell 2014; 158:69-83.

72 Muzumdar MD, Tasic B, Miyamichi K, Li L, Luo L. A global double-fluorescent Cre reporter mouse. Genesis 2007; 45:593605 .

(Supplementary information is linked to the online version of the paper on the Cell Research website.)

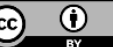

This work is licensed under a Creative Commons Attribution 4.0 Unported License. The images or other third party material in this article are included in the article's Creative Commons license, unless indicated otherwise in the credit line; if the material is not included under the Creative Commons license, users will need to obtain permission from the license holder to reproduce the material. To view a copy of this license, visit http:// creativecommons.org/licenses/by/4.0/

(C) The Author(s) 2017 